\title{
Análisis paleosismológico de la falla Acequión mediante perfiles topográficos de escarpa (Precordillera de San Juan, Argentina)
}

\author{
Paleoseismological analysis of the Acequión fault by means topographic scarpment profiles \\ (San Juan Precordillera, Argentina)
}

\author{
Bruno Colavitto ${ }^{1,2^{*}}$, Juan Manuel Alcacer ${ }^{1,2}$, Martín Rothis ${ }^{1,2}$, Federico Haro ${ }^{1,2}$, Pablo A. Blanc ${ }^{1,2}$, \\ Romina Onorato ${ }^{1,2}$, Laura Perucca ${ }^{1,2}$
}

${ }^{1}$ Gabinete de Neotectónica y Geomorfología. INGEO. Facultad de Ciencias Exactas, Físicas y Naturales. Universidad Nacional de San Juan, 5402, San Juan, Argentina.

${ }^{2}$ CIGEOBIO-UNSJ. Consejo Nacional de Investigaciones Científicas y Tecnológicas (CONICET), Av. Ignacio de La Roza y Meglioli s/n, 5400, San Juan, Argentina.

* Autor para correspondencia: (B. Colavitto) bcolavitto@gl.fcen.uba.ar

\section{Gómo citar este artículo:}

Colavitto, B., Alcacer, J. M., Rothis, M., Haro, F., Blanc, P. A., Onorato, R., Perucca, L., 2021, Análisis paleosismológico de la falla Acequión mediante perfiles topográficos de escarpa (Precordillera de San Juan, Argentina): Boletín de la Sociedad Geológica Mexicana, 73 (2), A090121. http://dx.doi.org/10.18268/ BSGM2021v73n2a090121

Manuscrito recibido: 13 de Septiembre de 2020 Manuscrito corregido: 20 de Diciembre de 2020 Manuscrito aceptado: 6 de Enero de 2020

La revisión por pares es responsabilidad de la Universidad Nacional Autónoma de México.

Este es un artículo Open Access bajo la licencia CC BY-NCSA (https://creativecommons.org/licenses/by-nc-sa/4.0/)

\section{RESUMEN}

Este trabajo se concentra en el extremo sur de la depresión Matagusanos-Maradona-Acequión entre la Precordillera Central y Oriental en la provincia de San Juan, Argentina. Se analizó una escarpa de falla asociada a la actividad cuaternaria de la falla inversa Acequión, de vergencia oriental. La misma muestra una longitud de aproximadamente $12 \mathrm{~km}$ con rumbo norte y un resalto vertical de hasta $\sim 12 \mathrm{~m}$ en algunos sectores. Tras un análisis geomorfológico con control de campo se la pudo clasificar como una escarpa flexural con crestas de presión con retrocabalgamientos. A partir de modelos digitales de elevación de $5 \mathrm{~m}$ de píxel, se levantaron 70 perfiles topográficos transversales a la estructura y se estudiaron estadísticamente los quiebres de pendiente que presenta la escarpa y los valores de desplazamiento vertical de la superficie. Considerando el tipo de escarpa estudiado, estos últimos parecieran brindar mejor información. En función de los resultados obtenidos es probable que hasta 4 o 5 eventos de Mw mínima de 6.57.1 hayan ocurrido en la falla Acequión durante los últimos $\sim 35 \mathrm{ka}$. Estos valores se aproximan a los obtenidos previamente por otros autores, aunque trabajos futuros de paleosismología de mayor detalle podrán confirmar la consistencia de la metodología aplicada. Adicionalmente, se realizó un levantamiento gravimétrico en el sector sur. La interpretación geofísica-geológica de la carta de anomalías residuales gravimétricas obtenidas, permitió definir la continuidad en subsuelo de al menos dos fallas de rumbo norte, sin evidencias de ruptura superficial. Estas estructuras pueden estar asociadas en profundidad a la falla Acequión y también estarían segmentando la bajada pedemontana oriental de la Precordillera Central.

Palabras clave: escarpa de falla, paleosismología, falla inversa, perfiles topográficos, neotectónica, gravimetría.

\section{ABSTRACT}

The present work focuses on the south tip of the more than $120 \mathrm{~km}$ long Matagusanos-Maradona-Acequión tectonic depression, located between the Eastern and Central Precordillera in San Fuan province, Argentina. A reverse fault scarp, associated with the Quaternary activity of the east vergent-Acequión fault was analyzed. This fault scarp has an average length of 12 $\mathrm{km}$ with a north trend and shows up to $\sim 12$ $m$ of throw. After a geomorphological mapping and fieldwork, it was typified as a flexural scarp with a back-thrust pressure ridge. Aided by digital elevation models with $5 \mathrm{~m}$ horizontal resolution, we constructed 70 transverse topographic profiles, from which slope breaks and vertical displacement values were extracted and statistically analyzed. Considering the fault scarp classification, the displacement values turned out to be more suitable for the paleoseismological analysis. According to our results, between 4 and 5 minimum $M w$ 6.5-7.1 earthquakes may have occurred in the Acequión fault since the last $\sim 35 \mathrm{ka}$. These values have some similarities to those previously obtained by other authors, although future and more detailed paleoseismological surveys should be carried out to test the methodology that was here applied. Additionally, a detailed gravimetric survey was conducted in the southern sector. The geophysical-geological interpretation of residual gravimetric anomalies showed that at least two more north-trending eastern structures parallel to Acequión fault affect the Central Precordillera eastern piedmont.

Keywords: fault scarp, paleoseismology, reverse fault, topographic profile, neotectonics, gravity data. 


\section{Introducción}

En este trabajo se presenta un estudio morfotectónico de una escarpa de falla inversa cuaternaria, con el objetivo de analizar las variaciones en su morfología y discutir qué implicancias tienen los cambios en su signatura superficial para el análisis de su potencial sismogénico. La zona de trabajo se ubica en el sur de la provincia de San Juan, en Argentina, entre los sistemas de Precordillera Central y Oriental (Figura 1). Esta unidad morfoestructural de primer orden se caracteriza por su larga y compleja historia geológica, que se remonta hasta el Paleozoico temprano (Ramos, 1988) y por concentrar gran parte de la actividad sísmica del territorio argentino (Costa et al., 2006, 2020). El carácter neotectónico de la Precordillera está condicionado en gran medida por el desarrollo del segmento de subducción horizontal pampeano de los Andes Centrales a partir de la subducción de la dorsal de Juan Fernández (Jordan et al., 1983; Cahill y Isacks, 1992; Smalley et al., 1993; Anderson et al., 2007).

Entre los sismos más importantes que han ocurrido en esta región de la Argentina se destacan aquellos ocurridos en 1894, 1944, y 1977 que causaron importantes daños en distintas localidades de la provincia (Costa et al., 2006; Perucca y Vargas, 2014). En este sentido, distintos trabajos han apuntado a la caracterización de las fuentes sismogénicas a partir de la identificación de indicadores morfotectónicos como escarpas de falla, anomalías del drenaje, desplazamientos laterales, etc., así como a partir de la aplicación de métodos geofísicos indirectos (Bastías et al., 1984; Siame et al., 1997; Terrizano et al., 2010, 2014; Perucca et al., 2014b, 2015; Alcacer Sánchez et al., 2018; Peri et al., 2020). Pese a ello, continúan siendo materia de debate las características de las fuentes sismogénicas en la Precordillera; siendo un limitante para su caracterización la difusa relación que existe entre eventos de elevada magnitud como los ocurridos en la provincia y la relevancia de las evidencias superficiales descriptas para las estructuras (Siame et al., 1997; Costa et al., 1999).
La falla Acequión se ubica al sur de la provincia de San Juan, en un sector conocido como Pampa del Acequión. Una escarpa de falla de rumbo norte de $12 \mathrm{~km}$ de longitud en niveles cuaternarios aluviales indica su actividad neotectónica. Las excelentes exposiciones naturales de esta estructura han motivado estudios que se enfocaron en el análisis de la deformación interna en depósitos lacustres y aluviales (Paredes y Perucca, 2000; Perucca y Moreiras, 2006, 2008; Perucca et al., 2009, 2014a). Dos eventos de magnitud superior a Mw 7.0 en los últimos 12-15 ka han sido identificados a partir de estos trabajos (Audemard et al., 2016). Sin embargo, aún resta hacer estudios paleosismológicos de mayor detalle y contar con más edades absolutas de las unidades involucradas en la deformación. Teniendo esto en cuenta, en este trabajo se plantea un enfoque diferente para constatar las conclusiones previas en torno a esta estructura y seguir contribuyendo a su caracterización como fuente sismogénica.

En sistemas transcurrentes de distintas partes del mundo la medición de los desplazamientos laterales de geoformas es un acercamiento válido para conocer la historia paleosísmica de una falla (Wallace, 1968; McGill y Sieh, 1991; Mériaux et al., 2004; Klinger et al., 2011; Zielke et al., 2012, 2015; Ren et al., 2016). Sin embargo, no ha sido tan exhaustivo el uso de técnicas similares para sistemas de fallamiento inverso (Carretier et al., 2002; Wei et al., 2019), en gran parte dado que revisten mayores complejidades relacionadas con la geometría de las fallas, las morfologías asociadas y el potencial de preservación de las topografías primarias (Philip et al., 1992; Audemard, 1999, 2003; Ollarves et al., 2006; McCalpin, 2009; Yu et al., 2010; López et al., 2011).

Se presenta un estudio geomorfológico de detalle a partir del análisis de imágenes satelitales y reconocimiento de campo. De esta manera se pretende caracterizar el tipo de escarpa de falla asociada a la falla Acequión para luego aplicar la metodología propuesta recientemente por Wei et al. (2019) para el estudio de fallas inversas. A partir de los valores obtenidos de este análisis se calcularon, 
mediante el uso de relaciones empíricas (Wells y Coppersmith, 1994), las magnitudes de los sismos potenciales. Adicionalmente, en el sector sur de la zona de estudio se realizaron relevamientos con un vehículo aéreo no tripulado (VANT o drone) para obtener datos topográficos de alta resolución junto con estudios gravimétricos de detalle. Con todo ello se pretende: 1) dilucidar el tipo de escarpa producido por la actividad neotectónica de la falla Acequión, 2) verificar la aplicabilidad de la metodología propuesta por Wei et al. (2019) y 3) constatar las propuestas anteriores en torno a la historia paleosísmica de la falla Acequión para que, con los nuevos datos obtenidos, se pueda mejorar la comprensión de esta estructura.

\section{Descripción del área de estudio}

\subsection{MARCO TECTÓNICO REGIONAL}

La Precordillera de San Juan, ubicada en los Andes Centrales, es una faja plegada y corrida compuesta principalmente por sedimentitas paleozoicas producto de la migración al este del frente orogénico durante el Mioceno, como consecuencia de una horizontalización de la losa oceánica desde los 18 Ma (e.g., Ramos, 1999; Ramos et al., 2002). Por sus características estructurales y litoestratigráficas se divide en tres sub-unidades, Occidental, Central y Oriental (Heim, 1952; Baldis y Chebli, 1969; Ortiz y Zambrano, 1981), pudiendo considerarse

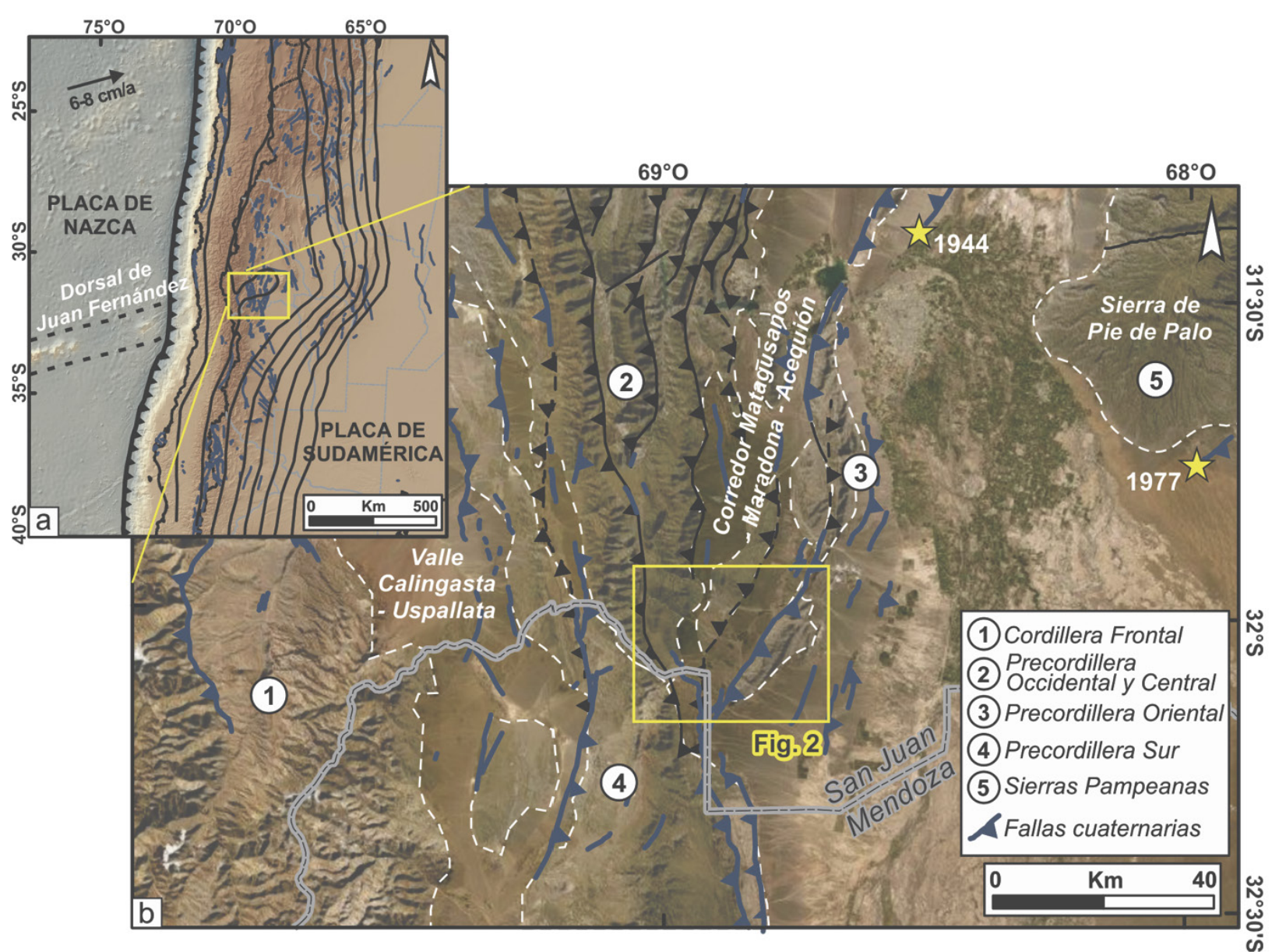

Figura 1 a) Ubicación del área de estudio en el contexto de los Andes Centrales de Argentina y Chile. Las líneas azules son fallas con actividad tectónica cuaternaria (Proyecto SARA, https://sara.openquake.org/). Las curvas de profundidad de la losa (Tassara y Echaurren, 2012) resaltan la Zona de Subducción Horizontal Pampeana donde se ubica el área de estudio (recuadro amarillo). b) Principales unidades morfoestructurales en la región de trabajo. Se indica con estrellas amarillas la ubicación de los epicentros de los sismos destructivos de 1944 y 1977. El recuadro amarillo muestra la ubicación del sector analizado en la Figura 2. 
también la Precordillera Sur, de características distintas y oblicua al rumbo de las anteriores (Cortés et al., 2006; Ahumada y Costa, 2009) (Figura 1b).

Los sistemas de Precordillera Central y Oriental se caracterizan por fallas inversas de rumbo norte con vergencias opuestas configurando una zona triangular de piel gruesa. La Precordillera Oriental muestra un estilo de piel gruesa y vergencia occidental, contrario al de la Precordillera Central (piel fina y vergencia oriental). Los cabalgamientos principales transportan la deformación al $\mathrm{E}$ imbricando las láminas antiguas sobre las más jóvenes y aumentando así la inclinación de las fallas antiguas al O (Giampaoli y Cegarra, 2003). La zona triangular que queda definida entre ambos sistemas precordilleranos (Zapata y Allmendinger, 1996; Ramos, 1997) coincide aproximadamente con el desarrollo de la depresión tectónica Matagusanos-Maradona-Acequión, de más de $120 \mathrm{~km}$ de largo (Figura 1). A lo largo de esta depresión, se han descrito numerosas estructuras activas durante el Cuaternario, en su mayoría fallas inversas de rumbo submeridional y altas tasas de deformación (Perucca, 1990; Paredes y Perucca, 2000; Siame et al., 2006; Perucca y Onorato, 2011; Perucca et al., 2013; Audemard et al., 2016; Lara et al., 2018).

\subsection{LA PAMPA DEL AGEQUIÓN Y LA FALLA AGEQUIÓN}

La Pampa del Acequión (Figura 2) se ubica en la porción austral de la depresión Matagusanos-Maradona-Acequión entre los ríos del Agua y Los Pozos (Perucca, 1990; Perucca et al., 2012, 2013). Limita al este con la sierra de Pedernal-Los Pozos, cuyo levantamiento está controlado por la falla Pedernal de vergencia occidental, y al oeste por las sierras de Las Osamentas y El Tontal, pertenecientes al dominio de la Precordillera Central (Figura 2). Esta zona se caracteriza por pliegues por propagación de falla imbricados y fallas fuera de secuencia, cuyo nivel de despegue se encontraría en el techo de las rocas cámbricas-ordovícicas (Giampaoli y Cegarra, 2003). En el sector norte de la zona de estudio afloran una serie de pliegues en rocas de la Formación Albarracín, de edad miocena, dando cuenta de la intensidad de la deformación andina en esta área.

La Pampa del Acequión es un pequeño valle ubicado entre los macizos montañosos antes mencionados, formados por areniscas y calizas paleozoicas y conglomerados neógenos. Esta depresión se encuentra cubierta por depósitos aluviales provenientes de la Precordillera Central, acarreados por el río Acequión y sus tributarios (Figura 2). La actividad tectónica, combinada con los ciclos glaciarios/interglaciarios controló el desarrollo de al menos dos niveles de terrazas aluviales de edad postglacial $(\sim 14 \mathrm{ka})$ que se encuentran cubriendo en discordancia secuencias de depósitos finos (Audemard et al., 2016). Estos últimos, de tonalidades claras, están formados por bancos de arena fina a limo, con escasas intercalaciones de arcilla o grava fina y se destacan por la abundante aparición de estructuras de deformación sedimentaria o sismitas (Paredes y Perucca, 2000). Estos afloramientos se pueden agrupar en dos (Perucca et al., 2009) o tres (Audemard et al., 2016) estadios en la evolución de un pequeño cuerpo lacustre tipo playa lake formado sobre la cara occidental de la Sierra de Pedernal, probablemente producto del endicamiento de la Boca del Acequión en dos ocasiones por avalanchas de roca (Perucca y Moreiras, 2008; Perucca et al. 2009) (Figura 2). Perucca et al. (2012) publicaron edades $\mathrm{C}^{14}$ de estos depósitos lacustres que sitúan al lago más antiguo en el estadio isotópico marino 3 ( $35 \mathrm{ka}$, MIS3) y al más reciente posterior al Último Máximo Glaciar $(\sim 14$ ka, UMG). Las estructuras de licuación halladas en estos extensos depósitos lacustres, entre las que destacan los diques de arena, fueron analizadas por Paredes y Perucca (2000), Perucca y Moreiras (2008) y Perucca et al. (2009) quienes interpretaron que las mismas se formaron tras la ocurrencia de entre dos y tres sismos durante el Pleistoceno tardío-Holoceno, adjudicados a la falla Cerro Salinas, ubicada al Este de la sierra de Pedernal, fuera del área de estudio (Figura 2).

En términos neotectónicos, en la zona de estudio se identificaron al menos tres fallas con actividad tectónica cuaternaria (Perucca et al., 2012, 
2013; Audemard et al., 2016): las fallas Acequión, La Chilca y Pedernal (Figura 2). En este trabajo se

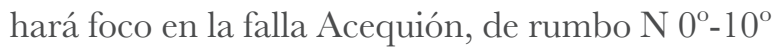
e inclinación aproximada de $26^{\circ}$ al $\mathrm{O}$ (Perucca et al., 2012). Esta estructura fue definida por Ahumada (2010) como falla Los Colorados e interpretada como la prolongación al sur de la falla Punta del Cerro de Giampaoli y Cegarra (2003). Afecta las terrazas aluviales y los depósitos lacustres antes descritos, estos últimos llegando a estar visiblemente inclinados al E en el bloque alto (Audemard et al., 2016). Según Perucca et al. (2012), forma una escarpa de falla flexural, múltiple con dos resaltes de $6 \mathrm{~m}$ y $1.8 \mathrm{~m}$ de altura asociados a bifurcaciones de la falla cuando alcanza la superficie. En exposiciones naturales de la falla Acequión, Audemard et al. (2016) describieron dos cuñas clásticas determinando que al menos dos eventos de $\mathrm{Mw}>7$ postUMG deben haber ocurrido en esta estructura.
Sin embargo, y en línea con la propuesta de Perucca et al. (2012), describieron esta falla como parte de un sistema más complejo que abarca una bifurcación menor de la falla hacia el bloque bajo y alcanzan a relacionar la falla Acequión con la propagación sur de la falla Cerro La Chilca, para la cual presentaron evidencias paleosísmicas de al menos cuatro eventos post-UMG, con un desplazamiento a lo largo de la falla por evento del orden de $1 \mathrm{~m}$ (Audemard et al., 2016).

\section{Metodología y datos empleados}

\subsection{ANÁLISIS MORFOTEGTÓNICO}

El análisis morfotectónico realizado incluye la caracterización geomorfológica de la Pampa del Acequión, en particular de la escarpa de la falla
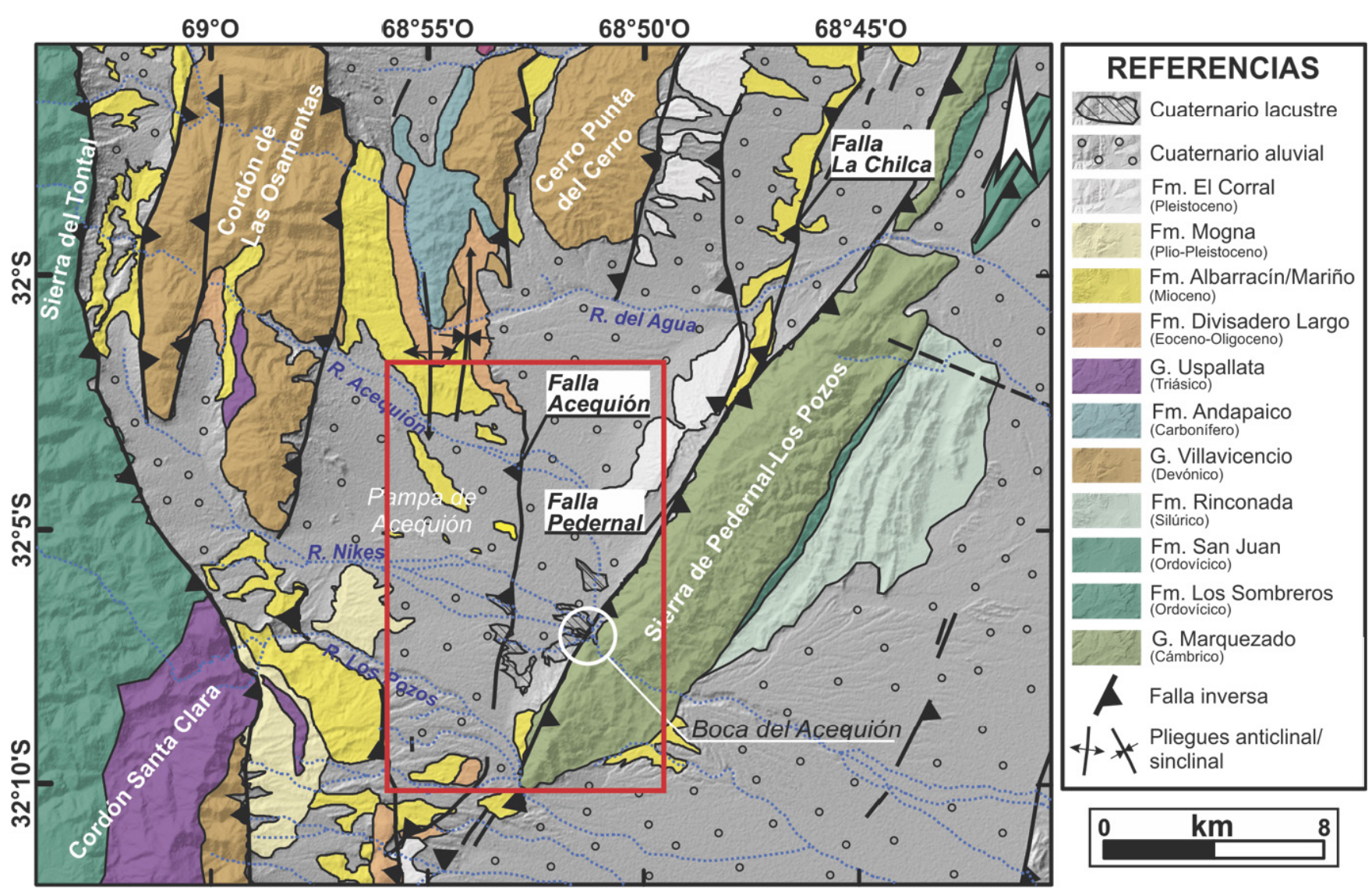

Figura 2 Principales estructuras y serranías que delimitan la Pampa de Acequión. Mapa geológico obtenido a partir de imágenes satelitales de Google Earth, ESRI y LandSat 7 ETM (combinación R7/5 G5/4 B3/1), de Giampaoli y Cegarra (2003), Perucca et al. (2012) y Pantano Zuñiga (2015). El recuadro rojo corresponde al sector analizado. 
Acequión. Mediante el uso de imágenes satelitales de Google Earth y LandSat 7 ETM se relevaron los principales rasgos geológicos de la zona de la Pampa del Acequión. Se refinaron mapas geológicos anteriores y se caracterizó la geomorfología del área, con la atención puesta en la identificación de aquellos rasgos morfológicos que pudieran actuar como indicadores de la deformación (Audemard, 1999, 2003; Ollarves et al., 2006; López et al., 2011; Burbank y Anderson, 2011). Durante el trabajo de campo se recorrieron los $12 \mathrm{~km}$ de extensión de la escarpa, buscando evidencia de deformación reciente de la cubierta aluvial y en las exposiciones naturales que forman los arroyos.

Para estudiar las características topográficas de la escarpa de falla se realizó un perfilaje topográfico, que ha demostrado ser un método útil para documentar la componente vertical del fallamiento y los distintos pulsos de construcción de una escarpa (Wallace, 1977; Bucknam y Anderson, 1979; Mayer, 1984; Andrews y Hanks, 1985; Arrowsmith et al., 1996; McCalpin, 2009; Audemard, 2009). La componente vertical de una escarpa registra la acumulación de sucesivos desplazamientos en el tiempo, producto de distintos eventos sísmicos en el caso de una escarpa de falla múltiple o compuesta (Wallace, 1977; Wei et al., 2019). Por su parte, los pulsos de construcción de una escarpa de falla inversa pueden estudiarse a partir de las variaciones en el gradiente del perfil de la escarpa (Carretier et al., 2002; Ewiak et al., 2015; Wei et al., 2019). Los perfiles se extrajeron del modelo digital de elevación -MDE- de $5 \mathrm{~m}$ de resolución horizontal provisto por el IGN (MDE-Ar obtenido por fotogrametría, https://www.ign.gob.ar). Mediante la Herramienta de Perfil de ArcGIS, se obtuvieron 70 perfiles transversales y un perfil paralelo al rumbo de la escarpa de falla Acequión (Figura 3). Los perfiles se realizaron de forma perpendicular a la escarpa de falla y en aquellos sectores donde la pendiente se encontraba lo menos obliterada posible (excluyéndose así sectores con erosión fluvial y/o modificados por la actividad humana).

Utilizando un algoritmo de MatLab creado para este propósito, se ajustó manualmente mediante una inspección visual, la pendiente del bloque alto y bloque bajo $(\delta)$, obteniendo así, la pendiente $(\beta)$ y la altura de escarpa $(\mathrm{H})$, el dislocamiento de la superficie (DV) y, adicionalmente, el número de quiebres en el gradiente (n) de la escarpa siguiendo la metodología propuesta por Wei et al. (2019) (Figura 3). La pendiente total de la escarpa se puede tomar como un proxy robusto sobre el grado de conservación de la morfología de la escarpa: para una misma falla y un mismo estilo de deformación, el ángulo de reposo de la escarpa es inversamente proporcional al grado de conservación de la escarpa: a mayor ángulo menos procesos superficiales afectaron el perfil, exceptuando aquellos casos donde la escarpa está visiblemente cortada por un curso fluvial (en esos casos se ven pendientes $\sim>20^{\circ}$ ). Esto es particularmente cierto para el caso de fallas normales (Ewiak et al., 2015) pero, bajo ciertas condiciones, puede extrapolarse lo mismo al análisis a escarpas de fallas inversas (Wei et al., 2019). Por su parte, la altura de la escarpa no fue utilizada y se midió un parámetro denominado dislocamiento o desplazamiento vertical de la superficie (DV). Según explican Yang et al. (2015), la altura de escarpa no debe tomarse en cuenta para caracterizar la falla ya que se cumple que $\mathrm{H}>>\mathrm{DV}$; mientras que, al no contar con un plano de falla unívoco para cada perfil, la medida del desplazamiento vertical utilizada es la más próxima a la medida del desplazamiento vertical de falla $(\mathrm{DRv})$. Siendo así se utilizó dicha medida para los cálculos, medida que según Wei et al. (2019) da cuenta del desplazamiento acumulado por la estructura en distintas superficies y al realizar un análisis estadístico debería mostrar agrupamientos en torno a distintos valores que representan distintos eventos. Los cambios en el gradiente a lo largo de cada perfil se graficaron siguiendo la metodología propuesta por Ewiak et al. (2015), y revisada por Wei et al. (2019), tomando distintas ventanas para segmentar el perfil: saltos de 3 píxeles ( $15 \mathrm{~m}$ ), 5 píxeles (25 m) y 7 píxeles ( 35 $\mathrm{m})$.

Finalmente, los datos obtenidos se procesaron en Excel evaluando las distribuciones estadísticas de los mismos y corroborando su valor como indicadores paleosísmicos. Dada la componente 

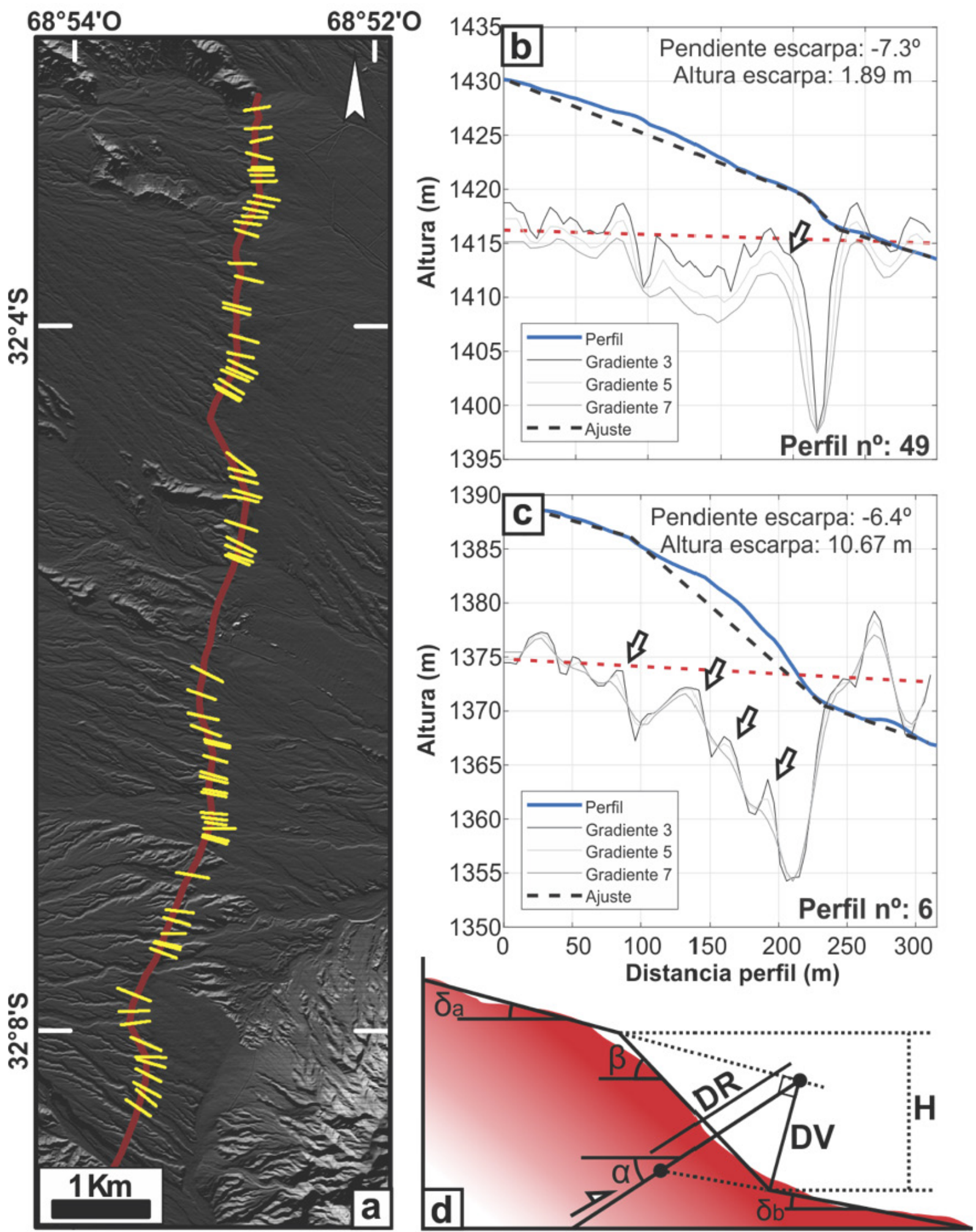

Figura 3 a) Perfiles topográficos realizados en la escarpa de la falla Acequión. b,c) Ejemplo de mediciones de la geometría de la escarpa, realizadas con el algoritmo de MatLab. Se indican con flechas los quiebres de pendiente. d) Esquema de parámetros medidos, modificado de Yang et al. (2015). H: altura de escarpa; DV: desplazamiento o dislocamiento vertical de la superficie; DR: desplazamiento real, a lo largo de la falla; $\alpha$ : inclinación de falla; $\beta$ : pendiente promedio de escarpa; $\delta$ a,b: pendiente de campo lejano (far field slope) bloque alto (a) y bajo (b). 
manual de este procedimiento, se repitió el proceso en tres oportunidades a los fines de mejorar la confiabilidad de los datos. En este trabajo se muestran resultados parciales para uno de estos y los resultados finales obtenidos en cada uno de los experimentos.

\subsection{GRAVIMETRÍA Y PERFIL TOPOGRÁFICO DE DETALLE}

Para el análisis geofísico local se realizó un perfil gravimétrico de detalle de orientación O-E, a fin de atravesar en forma perpendicular las estructuras geológicas de interés. El perfil se efectuó con una separación de punto-estación de 200 m y con una precisión de $0.5 \mathrm{mGal}$, empleándose para su medición un gravímetro SCINTREX CG-5 (\#40484).

Las anomalías de gravedad fueron calculadas empleando la gravedad teórica de acuerdo con la expresión de Somigliana (1930) para el elipsoide de referencia de 1980 (GRS80; Geodetic Reference System, 1980). Se consideró un gradiente vertical de gravedad normal para la corrección de aire libre $(\mathrm{CAA}=0.3086 \mathrm{~h})$ y un modelo de losa infinita para la corrección de Bouguer simple $(\mathrm{CB}=0.0419$ $\sigma \mathrm{t} h$ ), siendo h la altura del punto-estación en metros sobre el nivel medio del mar. De acuerdo con lo propuesto por Hinze (2003), se consideró una densidad de $2.67 \mathrm{~g} / \mathrm{cm}^{3}$ para las rocas correspondientes a la corteza superior. En el cálculo de la anomalía de Bouguer Completa, se aplicó la corrección de terreno empleando los algoritmos desarrollados por Kane (1962) y Nagy (1966); en el cómputo se consideró un modelo de elevación local $(10$ x 10 m) y un modelo de elevación regional (100 x $100 \mathrm{~m})$, siendo ambos modelos obtenidos desde el modelo digital de elevación global STRM (Shuttle Radar Topography Mission) (Farr et al., 2007).

Por otra parte, a los fines de obtener datos topográficos de mayor precisión en la zona donde se efectuó el relevamiento gravimétrico, se realizó un vuelo con un vehículo aéreo no tripulado (drone). Se cubrió un área de 400 x 600 m, obteniéndose un modelo digital de alta resolución de $0.1 \mathrm{~m}$ de píxel mediante el software Agisoft Photoscan.

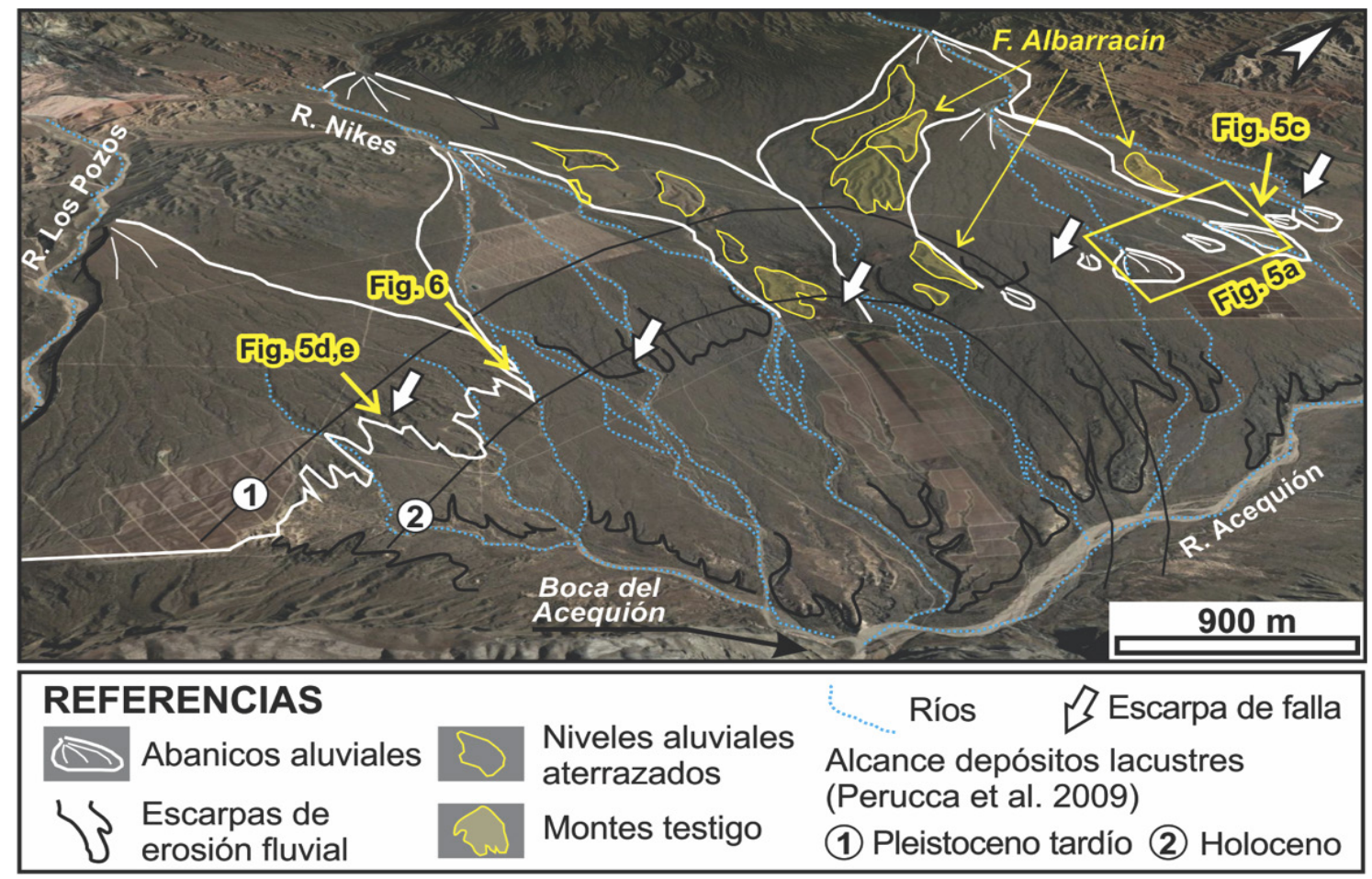

Figura 4 Principales rasgos geomorfológicos identificados en la zona de estudio. Entre otros elementos, se señalan los abanicos aluviales activos e inactivos (entre estos últimos dominan tres grandes abanicos correspondientes a los ríos Acequión, Nikes y Los Pozos). Se incluye también la extensión de los depósitos lacustres siguiendo la propuesta de Perucca et al. (2009) y se incluye la ubicación de los elementos de las Figuras 5 y 6. 


\section{Resultados}

\subsection{EVIDENGIAS DE GAMPO}

La escarpa de la falla Acequión resalta en imágenes satelitales y en los modelos digitales de elevación como un rasgo lineal de aproximadamente $12 \mathrm{~km}$ de longitud de rumbo norte. Está labrada sobre depósitos de abanicos aluviales, asignados al Pleistoceno superior a Holoceno, formados por gravas angulosas a subredondeadas, con intercalaciones de lentes arenosas. Estos depósitos, provenientes de los ríos Los Pozos, Nikes y Acequión (Figura 4), son parte de al menos dos generaciones de antiguos abanicos aluviales que actualmente se encuentran inactivos, como dan cuenta las distintas evidencias de erosión fluvial presentes en el área, coincidiendo con los dos grandes niveles de terrazas aluviales mencionados en la sección 2.2 (Figura 4). También se aprecia un drenaje distributario al pie de la escarpa en su sector norte, producto del resalto topográfico (Figura 4). Este patrón distributario no solo ayuda a trazar el rumbo de la estructura, sino que el mismo puede ser indicativo de la deformación reciente (Audemard, 1999; Ollarves et al., 2006). Por sectores se asocia con sutiles cambios de diseño en los ríos, aguas arriba de la escarpa, que aumentan su sinuosidad y confluyen entre sí volviéndose un único canal para incidirla y, luego de atravesarla, recuperan el patrón distributario (Figura 5a). Por otra parte, al menos 3 niveles de terrazas aluviales se identificaron en el bloque alto, en las proximidades de la escarpa en distintos segmentos (Figuras 5b y $5 \mathrm{~d}$ ).

En líneas generales, la escarpa de falla parece mostrar dos comportamientos distintos. Un sector sur donde el resalto topográfico es marcado (Figura 5c) y un sector norte, donde la pendiente de escarpa es más tendida y su expresión superficial es más dificil de observar en el campo, incluso muchas veces al estar cubierta por la vegetación baja típica de la zona (Figura 5b). El cambio entre
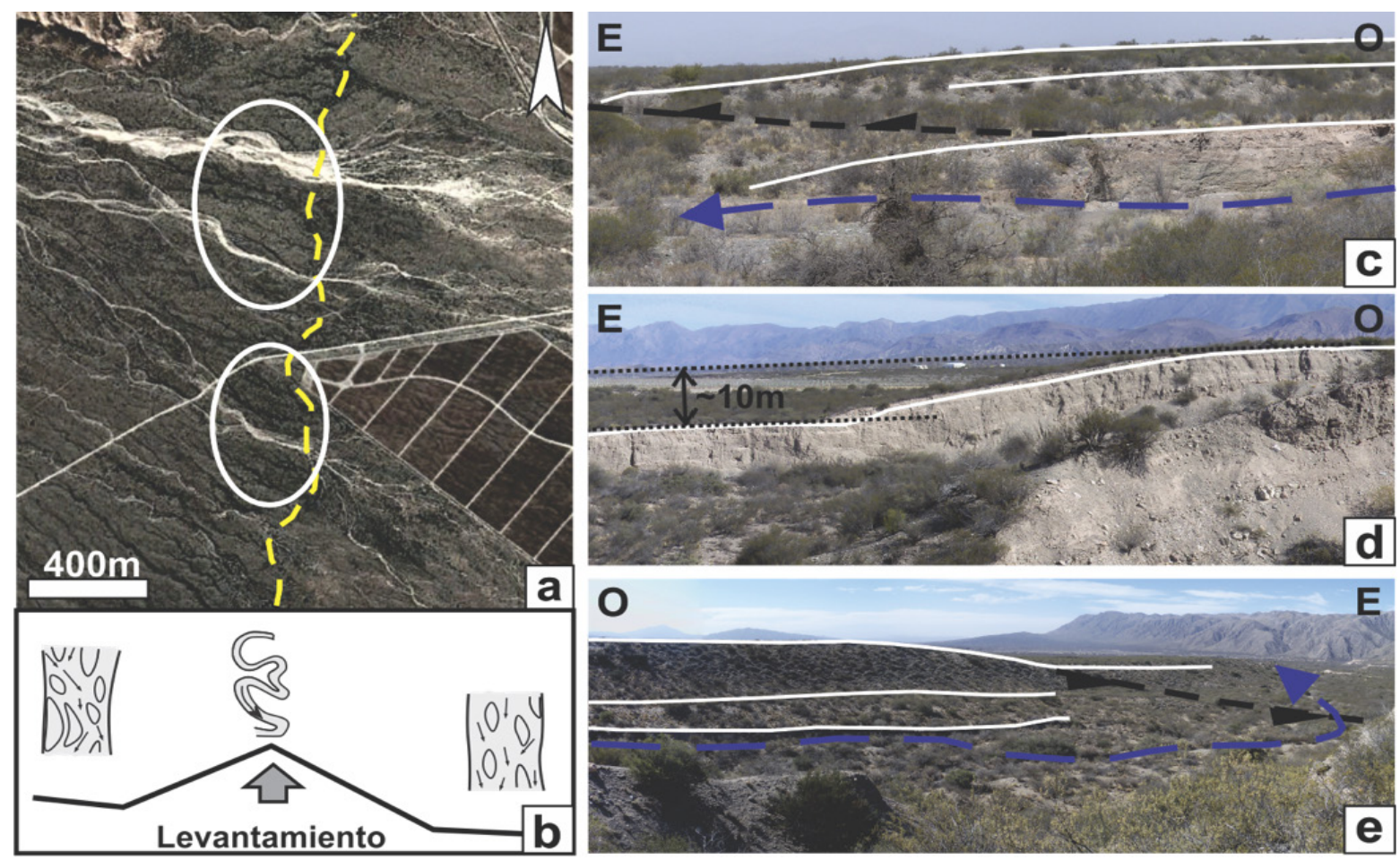

Figura 5 Indicadores morfotectónicos. a) Imagen de Google Earth con ajuste de tonalidades para resaltar los cambios en el drenaje asociados a la escarpa. b) Modelo simplificado de Litchfield et al. (2003) de cambios en el hábito de ríos entrelazados en una zona de levantamiento. c) Terrazas aluviales en el bloque alto de la falla (sector norte). d) Foto de campo en el sector sur donde se aprecia la escarpa flexural asociada a la falla Acequión. Nótese el contraste con la signatura morfológica de la misma en el sector norte. e) Terrazas aluviales en el bloque alto de la falla (sector sur). Véanse las ubicaciones en Figura 4. 
estos dos sectores coincide con la zona interfluvial de los ríos Nikes y Acequión (Figura 4). En este mismo sector se preservan montes testigos formados por rocas neógenas de la Formación Albarracín, sobre los que la traza de la escarpa también se puede continuar.

En el sector sur afloran unidades lacustres correspondientes al Pleistoceno superior-Holoceno (Figuras 4 y 6). Estas unidades, previamente descritas por otros autores (véase sección 2.2), están formadas por niveles de limo y arena fina, con algunas lentes areno-gravosas hacia el techo (Figura 6b). Durante el trabajo de campo, no solo se observaron en estos depósitos estructuras menores de deformación sinsedimentaria, sino también se encontró que los mismos guardan el registro de la deformación acumulada por la falla Acequión: en el bloque alto, los depósitos se encuentran inclinando entre $13^{\circ}$ y $24^{\circ}$ al ESE (Figura 6c), mientras que en el bloque bajo están en posición subhorizontal (Figura 6b).

Como se aprecia en la Figura 7, la erosión fluvial ha producido verdaderas trincheras naturales. $\mathrm{Al}$ norte, donde, como se dijo, las alturas de escarpa son menores, la incisión sólo revela parcialmente la estructura. En un afloramiento en este sector se puede observar una falla de vergencia oriental que afecta conglomerados de un nivel aluvial reciente (Figura 7b). Hacia el sur, sobre la margen norte del río Nikes, se pudieron identificar dos fallas inversas con inclinaciones al E, contrario a lo esperado en una falla con vergencia andina (Figura 7c). En este caso, las estructuras coinciden espacialmente con un resalto topográfico de

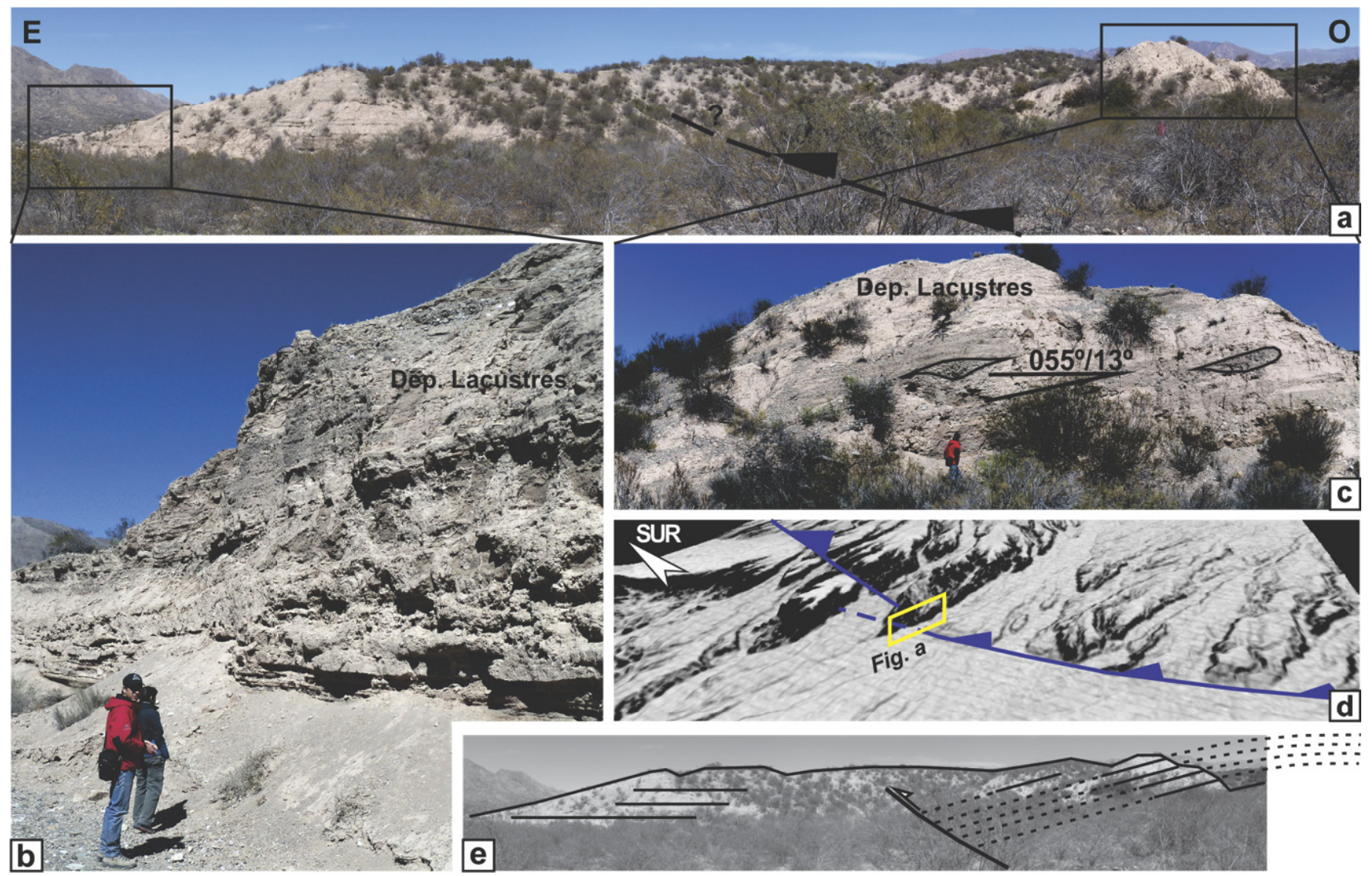

Figura 6 Depósitos lacustres del Pleistoceno superior - Holoceno, sector sur. a) Vista general del afloramiento (véase ubicación en Figura 4). b) Bancos subhorizontales dominados por arenas finas a medias y limos. c) Los mismos depósitos lacustres basculados al ESE, se señalan algunas lentes gravosas intercaladas. d) Modelo digital de elevación tomado del IGN con filtro por pendiente para resaltar la escarpa. e) Propuesta de esquema estructural. 
aproximadamente 4 a $5 \mathrm{~m}$ de altura y la deformación alcanza hasta los niveles más superficiales, a excepción de la cubierta de suelo. Por último, en el extremo sur, unidades aluviales procedentes del río Los Pozos se hallan fuertemente deformadas por una estructura de vergencia oriental, que también fuera mencionada por Audemard et al. (2016): se desarrolla un suave plegamiento sobre los niveles aluviales con una altura del orden de los 9 a 10 $\mathrm{m}$. Una falla de vergencia oriental afecta el frente de dicho plegamiento coincidente con el pie de la escarpa y, además, desarrolla un pequeño retrocabalgamiento (Figura 7d).

\subsection{ANÁLISIS MORFOTEGTÓNICO}

El perfilaje realizado permitió evaluar cambios en la morfología de la escarpa. De norte a sur se observa una disminución en la topografía, distinguiéndose tres segmentos, uno con mayores alturas al norte, un sector central con bajas alturas coincidente con los cursos del abanico aluvial que forma el río Nikes y un sector sur donde se ve un incipiente aumento de la topografía, coincidentemente con los afloramientos de depósitos lacustres del Pleistoceno superior-Holoceno y los relictos de un antiguo abanico aluvial procedente del río
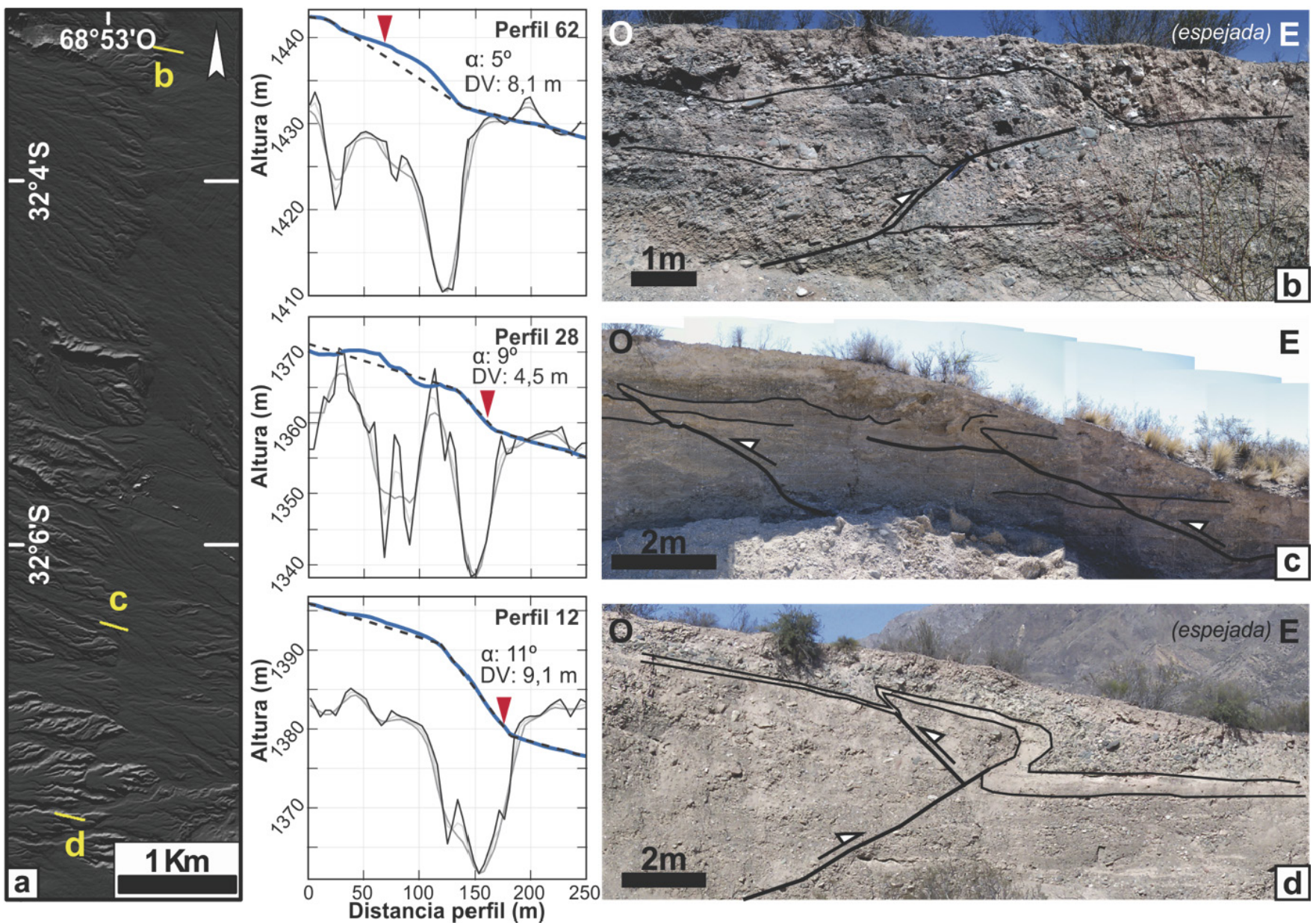

Figura 7 Trincheras naturales con exposición de estructuras asociadas a la falla Acequión. a) Modelo digital de elevación con la ubicación de las trincheras y perfiles topográficos de cada locación. b) Afloramiento al norte con pendiente tendida, donde se ve una estructura en la parte distal al pie de escarpa afectando depósitos aluviales de una terraza baja. c) Afloramientos en la margen norte del río Nikes. Dos estructuras de vergencia oeste deforman secuencias aluviales que sobreyacen depósitos lacustres inclinando al E. d) Afloramiento en el sector sur de la Pampa de Acequión. Dos cabalgamientos y un retrocabalgamiento conjugado deforman el frente de la escarpa de falla Acequión. b) y d) se espejaron para mostrar la misma orientación entre los afloramientos. Las flechas rojas sobre el perfil indican ubicación aproximada del afloramiento. 


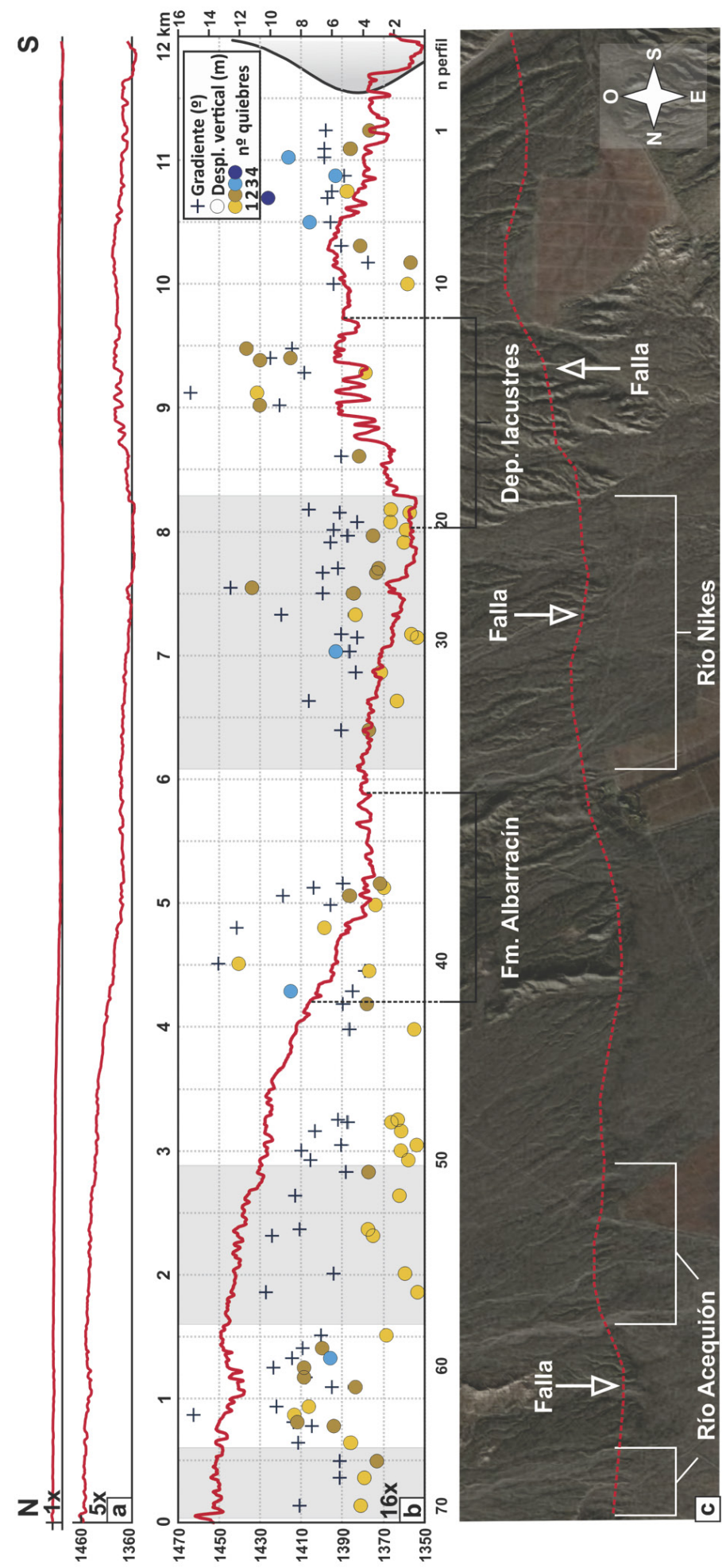

Figura 8 Perfil longitudinal de escarpa. a) Perfil longitudinal sin exageración vertical y con exageración vertical de $5 x$. b) Perfil longitudinal exagerado verticalmente $(16 \mathrm{x})$ con los datos obtenidos de los perfiles transversales sobre impuestos. En la margen derecha se aprecia la distribución estadística de los valores de desplazamientos verticales medidos. c) Imagen de Google Earth (espejada), sobre la que se muestra la traza del perfil longitudinal y algunos elementos morfológicos relevantes. Se muestra además la ubicación de los afloramientos de fallas (Figura 7). 
Los Pozos (Figura 8). Como se puede observar en el perfil topográfico con exageración vertical, el relieve irregular en la zona sur indica un mayor grado de incisión fluvial (Figura 8). En la figura 8, también se muestra la distribución espacial a lo largo de la escarpa del gradiente de la escarpa, el desplazamiento vertical y la cantidad de quiebres medidos en cada perfil transversal (Figura 8). Coincidentemente con el sector antes mencionado, los mayores desplazamientos verticales de la escarpa también fueron registrados en el sector sur, con la excepción de algunos perfiles del sector central, perfiles 26 y 40, medidos sobre una superficie aluvial antigua y sobre niveles de la Formación Albarracín (Mioceno superior) respectivamente.

Los perfiles transversales se midieron ajustando las pendientes de campo lejano del bloque alto y bloque bajo (Figura 3). Es importante considerar que no haya grandes diferencias entre las superficies agua arriba y abajo de la escarpa ya que de otra manera no sería confiable utilizar la superficie como indicativo de los desplazamientos. En promedio, esta diferencia fue menor a $1^{\circ}\left(\sim 0.75^{\circ}\right)$ mientras que la máxima diferencia fue de $2^{\circ} \mathrm{y}$ estuvo localizada en los perfiles 47 a 52. En este segmento la pendiente de campo lejano superior mostró un suave plegamiento de la superficie coincidente con el sector donde se observaron anomalías del drenaje (Figura 5a).
En cuanto al gradiente de la escarpa, en todos los casos no superó los $15^{\circ}$, y en más del $50 \%$ de las veces se registró un gradiente de entre $3^{\circ} \mathrm{y}$ $9^{\circ}$. Estos valores de pendiente son relativamente bajos, pero destacan de la pendiente registrada en las superficies aluviales tanto agua arriba como agua abajo. En los gráficos estadísticos se vio que no existe una correlación confiable entre la pendiente de la escarpa y el desplazamiento vertical observado y, si la hubiera, sería una correlación positiva (Figura 9a). Lo mismo se comprobó para la pendiente y el número de saltos de gradiente en el perfil de la escarpa (Figura 9b). Sin embargo, al cruzar los datos de desplazamientos verticales registrados con la cantidad de cambios de pendiente identificados en cada perfil sí se pudo corroborar la existencia de una tendencia (Figura 9c). Así es que, según se desprende del diagrama estadístico, aquellos perfiles de escarpa con un solo quiebre de pendiente (i.e. un evento) mostraron en su mayoría desplazamientos verticales de entre 2 y $4 \mathrm{~m}$, mientras que a medida que aumentan la cantidad de quiebres reconocidos los desplazamientos verticales medidos fueron mayores (entre 4 y $8 \mathrm{~m}$ para $\mathrm{n}=2$, entre 6 y $9 \mathrm{~m}$ para $\mathrm{n}=3$ y mayores a 10 $\mathrm{m}$ para los perfiles con $\mathrm{n}=4$ quiebres) (Figura 9c).

Se intentaron agrupar los datos obtenidos del perfilaje y analizar si los mismos pueden considerarse como indicadores paleosísmicos (sección
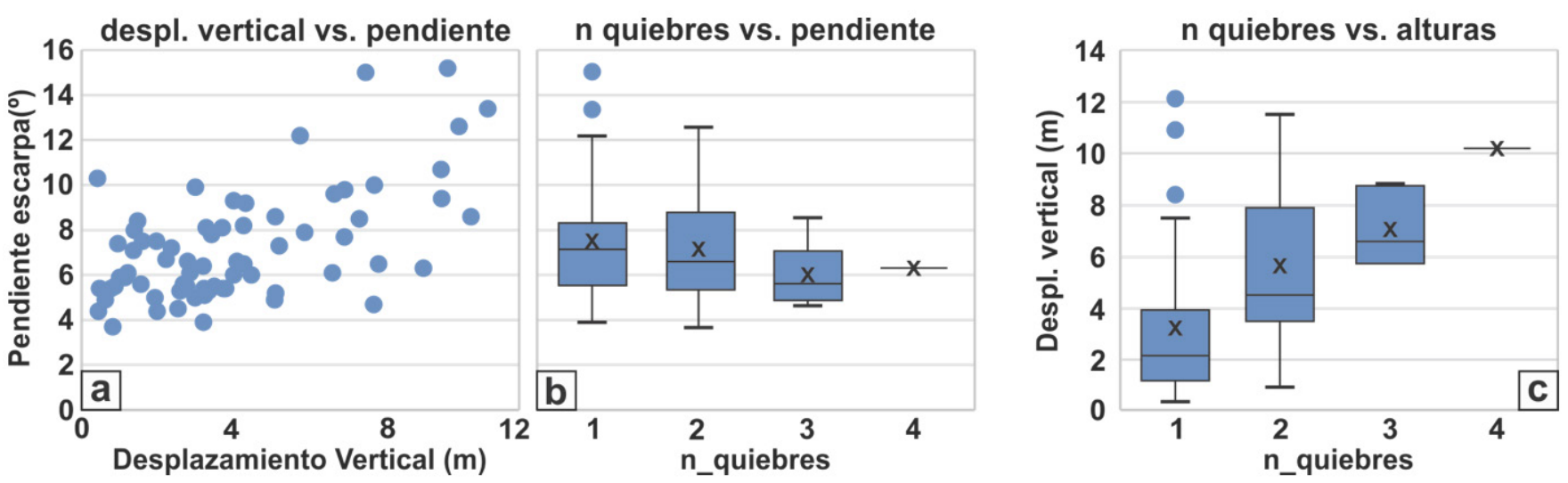

Figura 9 Gráficos estadísticos. a) Correlación entre los valores de desplazamiento vertical y pendiente de escarpa medidos para cada perfil. b) Diagrama de caja de la distribución de las pendientes de escarpa para cada $\mathbf{n}$ de quiebres de pendiente. c) Diagrama de caja de la distribución de los desplazamientos verticales medidos para cada $\mathbf{n}$ quiebres de pendiente identificados. 
Tabla 1. Análisis estadístico. Se muestran los valores obtenidos para la distribución de los desplazamientos verticales agrupados según quiebres de pendiente o según picos máximos (Sección 3.1).

\begin{tabular}{|c|c|c|c|c|c|c|c|c|c|c|}
\hline & Total & \multicolumn{4}{|c|}{ Por quiebres de pendiente } & \multicolumn{5}{|c|}{ Por desplazamiento vertical } \\
\hline & - & $\mathrm{n}=1$ & $\mathrm{n}=2$ & $\mathrm{n}=3$ & $\mathrm{n}=4$ & $\mathrm{~h}<2.5$ & $2.5<\mathrm{h}<5$ & $5<\mathrm{h}<7$ & $7<\mathrm{h}<9$ & $10<\mathrm{h}<13$ \\
\hline Media & 4.6 & 3.2 & 5.7 & 7 & $10.16^{*}$ & 1.3 & 3.7 & 5.9 & 8.2 & 11 \\
\hline$\pm \sigma$ & 3,1 & 2.7 & 2.9 & 1.4 & $0.012 *$ & 0.5 & 0.7 & 0.5 & 0.5 & 0.012 \\
\hline Moda & 3.6 & 2.2 & 3.6 & 5.8 & & 0.5 & 3.6 & 5.8 & 7.5 & 10.7 \\
\hline Mediana & 3.7 & 2.2 & 4.6 & 6.6 & $10.16^{*}$ & 1.3 & 3.7 & 5,8 & 8.3 & 10.9 \\
\hline Asimetría & 0.8 & 1.7 & 0.8 & 0.4 & & 0.2 & 0.09 & -0.06 & -0.14 & 0.4 \\
\hline Pruebas & & \multicolumn{4}{|c|}{ Por quiebres de pendiente } & \multicolumn{5}{|c|}{ Por desplazamiento vertical } \\
\hline Media & - & 2.2 & 3.6 & 7 & 10.7 & 1.3 & 3.5 & 5.7 & 8.1 & 10.7 \\
\hline$\pm \sigma$ & - & 0.8 & 0.4 & 1.2 & 0.7 & 0.4 & 0.25 & 0.35 & 0.4 & 0.6 \\
\hline
\end{tabular}

3.1). Partiendo de la distribución observada entre el número de quiebres y los desplazamientos verticales medidos (Figura 9c), se construyó un histograma de frecuencias agrupando los datos según el número de cambios de pendiente observados (Figura 10a). Las cuatro clases construidas tienen distribuciones con características distintas. Las muestras con uno y dos saltos de pendiente poseen curvas de distribución asimétrica positiva (con 1.7 y 0.8 de coeficientes de asimetría, respectivamente) lo que hace que media, mediana y moda estén desfasadas (Tabla 1). Sin embargo, es de esperar que procesos superficiales hayan eliminado algún quiebre de pendiente en aquellas escarpas con mayor desplazamiento vertical acumulado. De ser así, las clases de $n=1$ y $n=2$ quiebres de pendiente con valores altos de desplazamiento vertical estarían sobre-representadas. Teniendo esto en cuenta se aproximó cada clase a distribuciones normales, tomando como parámetros las medias y desviaciones estándar para cada grupo y, en el caso de las distribuciones asimétricas las modas, y se ajustaron las desviaciones estándar manualmente (Tabla 1). De esta manera se pondera la dispersión antes mencionada.

Se obtuvieron así 4 valores de desplazamientos verticales acumulados distintos en el caso de las clases distinguidas por número de quiebres en la pendiente y cinco valores de desplazamiento vertical acumulado para las clases construidas a partir de la distribución total de DV (Figura 10). A partir de ambas clasificaciones, resalta la existencia de un pico alrededor de $3.5 \pm 0.35 \mathrm{~m}$ y un pico en 10.7 $\pm 0.6 \mathrm{~m}$ (Tabla 1, Figura 10). El valor mínimo de $1.3 \pm 0.4 \mathrm{~m}$ obtenido por el agrupamiento según DV muestra una desviación estándar menor comparado con el valor mínimo para la clase $n=1$, lo cual da cuenta de la dispersión de los datos ya mencionada (Tabla 1, Figura 10). La mayor diferencia entre ambos modos de agrupamiento aparece para los desplazamientos verticales que ocurren entre 5 y $9 \mathrm{~m}$ aproximadamente donde, 
como se podía prever en la figura 9c, se registraron perfiles con 1, 2 y hasta 3 quiebres de pendiente. En este caso, siguiendo la clasificación por cantidad de quiebres en las pendientes de escarpa existiría un único valor $(7 \pm 1.2 \mathrm{~m})$; mientras que el agrupamiento de alturas realizado distingue dos valores: $5.7 \pm 0.35 \mathrm{~m}$ y $8.1 \pm 0.4 \mathrm{~m}$.

\subsection{ANÁLISIS GRAVIMÉTRICO}

El sector sur reviste cierta particularidad ya que no solo allí la escarpa de falla se encuentra levemente desfasada al oeste respecto a su traza completa, sino que existe además una marcada incisión fluvial dando lugar al menos a dos escalones (perfil topográfico en Figura 11c). En este sector, la Pampa del Acequión comienza a cerrarse y posiblemente exista interacción con estructuras de la Precordillera Sur y de la sierra de Pedernal, tal como lo expresaron Audemard et al. (2016) quienes demostraron esta interacción a pocas centenas de metros al sur de la quebrada del río Acequión. Para estudiar ello se realizó un perfilaje gravimétrico detallado (ver sección 3.2) coincidente con la ubicación del afloramiento de falla de la figura $7 \mathrm{c}$.
Los resultados del relevamiento se muestran en la figura 11. El análisis de los perfiles de anomalías (Figura 11c) muestra variaciones netamente marcadas en la tendencia general de las curvas de anomalía. Estos resaltos pueden ser resultado de contrastes de densidad en el subsuelo. A su vez, en análisis de las cartas de anomalías (Figura 11d) exhibe inflexiones en los valores de anomalías esperados de acuerdo con la pendiente regional existente en el área de estudio: estos gradientes resultan de la alternancia de máximos y mínimos relativos en pocos metros de desplazamiento horizontal, lo cual sugiere la disposición en el subsuelo de rocas de diferente naturaleza. Estos rasgos podrían corresponder a la continuidad espacial de dos fallas con orientación norte, paralelas a la falla Acequión, que también podrían mostrar actividad neotectónica, ocasionando una segmentación del basamento bajo el área de estudio, afectando a la vez a los depósitos pleistoceno tardío-holocenos. Este tipo de estructuras podría corresponderse con bifurcaciones de una estructura profunda. Por otra parte, levemente más al sur, una pequeña escarpa lineal labrada sobre una terraza fluvial del río Los Pozos podría ser interpretada como la prolongación al sur de alguna de estas estructuras (Figura 11a).
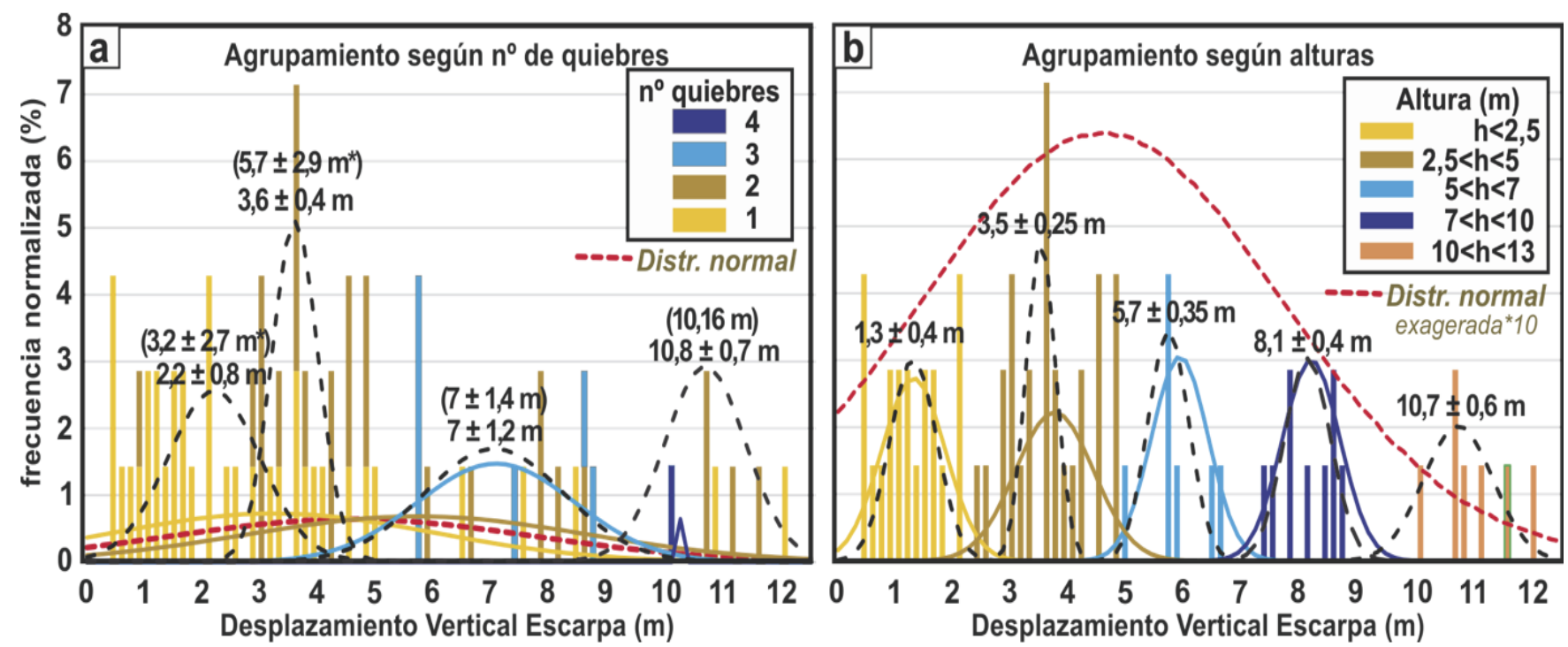

Figura 10 Distribuciones de frecuencia normalizada para los valores de desplazamiento vertical de superficie. a) Agrupamiento por número de quiebres de pendiente identificados. Los valores entre paréntesis corresponden con los parámetros de la distribución normal obtenidos por ajuste automático de cada clase. b) Agrupamiento según picos máximos de desplazamientos verticales. Tanto en este caso como en el anterior se muestran los valores que resultan del ajuste manual de las distribuciones (valor medio y desviación estándar para cada caso). La línea roja punteada en cada gráfico representa la curva de distribución normal para todo el conjunto de datos. 

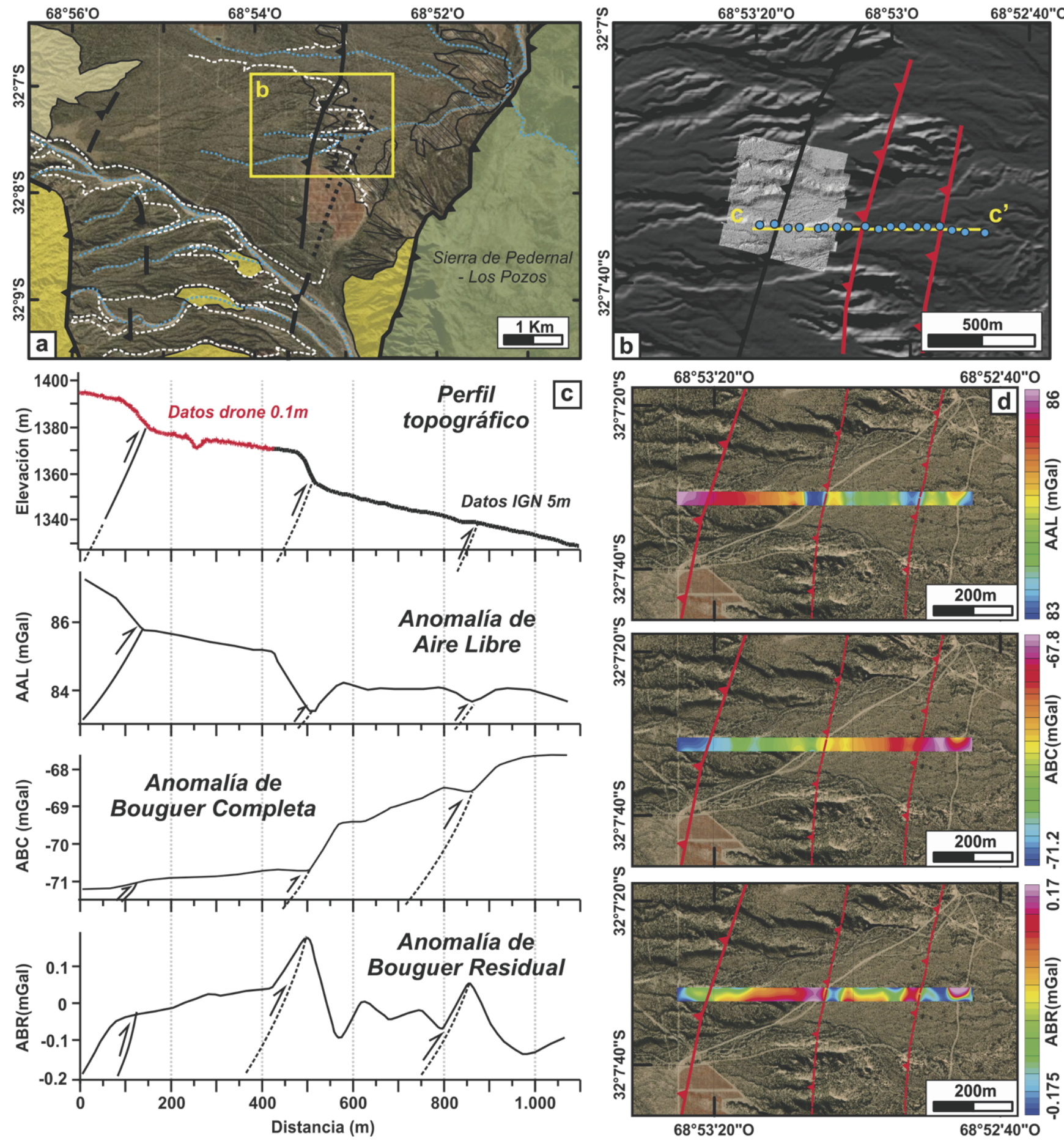

Figura 11 Resultados del análisis gravimétrico. a) Mapa geológico simplificado del área donde se realizó el relevamiento; en trazos punteados se sugiere la prolongación de algunas estructuras del área de trabajo. b) Modelo digital de elevación indicando la ubicación de las estaciones de medición del gravímetro a lo largo de la traza del perfil; el DEM corresponde con aquel obtenido por drone (0.1 $\mathrm{m}$ de píxel) sobreimpuesto al DEM del IGN de $5 \mathrm{~m}$ de píxel. c) Perfil topográfico obtenido del modelo digital de elevación calculado a partir de datos drone y del IGN; perfil de anomalía de Aire Libre; Bouguer Completa y Bouguer Residual cálculos a partir del perfil gravimétrico de detalle. d) Cartas de anomalías de Aire Libre, Bouguer completa y Bouguer Residual obtenidas mediante grillado por método de mínima curvatura. 


\section{Discusión}

\subsection{TIPO DE ESGARPA Y ESTRUGTURAS ASOGIADAS}

Se han presentado evidencias de campo de la actividad reciente de la falla Acequión y su impronta morfológica, correspondiente con una escarpa de falla inversa de $12 \mathrm{~km}$ de longitud. Incisiones transversales a la escarpa producto de la erosión vertical de arroyos efímeros permiten observar en algunos sectores el comportamiento de la estructura. En dos de los tres cortes transversales se observaron fallas de vergencia oriental, una de ellas asociada a un pequeño retrocabalgamiento próximo a la superficie (Figura 7). En el corte correspondiente al río Nikes se observaron dos cabalgamientos de vergencia occidental, cuya inclinación es casi paralela a la inclinación de la escarpa (Figura 7). En el afloramiento del sector sur, además, se puede observar con claridad la flexura en la superficie terrestre, que de forma indirecta también puede interpretarse a partir de las anomalías de drenaje observadas en el sector norte (Figura 5). A su vez, en distintos afloramientos se observaron depósitos lacustres con inclinaciones en el orden de los $13^{\circ}$ a $24^{\circ}$ al ESE; pudiendo corresponder, esta inclinación, al limbo frontal de un suave plegamiento (Figuras 6e y 12). Con todo esto es posible, y preciso, clasificar el tipo de escarpa de falla estudiada, a los fines de poder enmarcar los resultados que se obtengan en el modelo sismogénico (Yang et al., 2015). Siguiendo el esquema propuesto en la figura 12, puede considerarse que la geometría de las estructuras observadas en superficie sea parte de una escarpa flexural con cresta de presión con retrocabalgamientos, combinando dos de los modelos propuestos por Yu et al. (2010).

En cuanto a si se trata o no de una escarpa de falla múltiple, como han propuesto Perucca et al. (2012) si bien el modelo propuesto contempla la existencia de fallas menores desarrolladas en la cresta de presión, la estructura sismogénica asociada a la escarpa estudiada parecería unívoca. Por el contrario, en el sector sur, tanto el perfil topográfico como el relevamiento gravimétrico (Figura 11) sí sugieren la existencia de al menos dos estructuras más, hacia el este. Sin embargo, en este caso, cada una de estas estructuras habría desarrollado resaltos topográficos independientes (Figura 12), si bien no se descarta que compartan

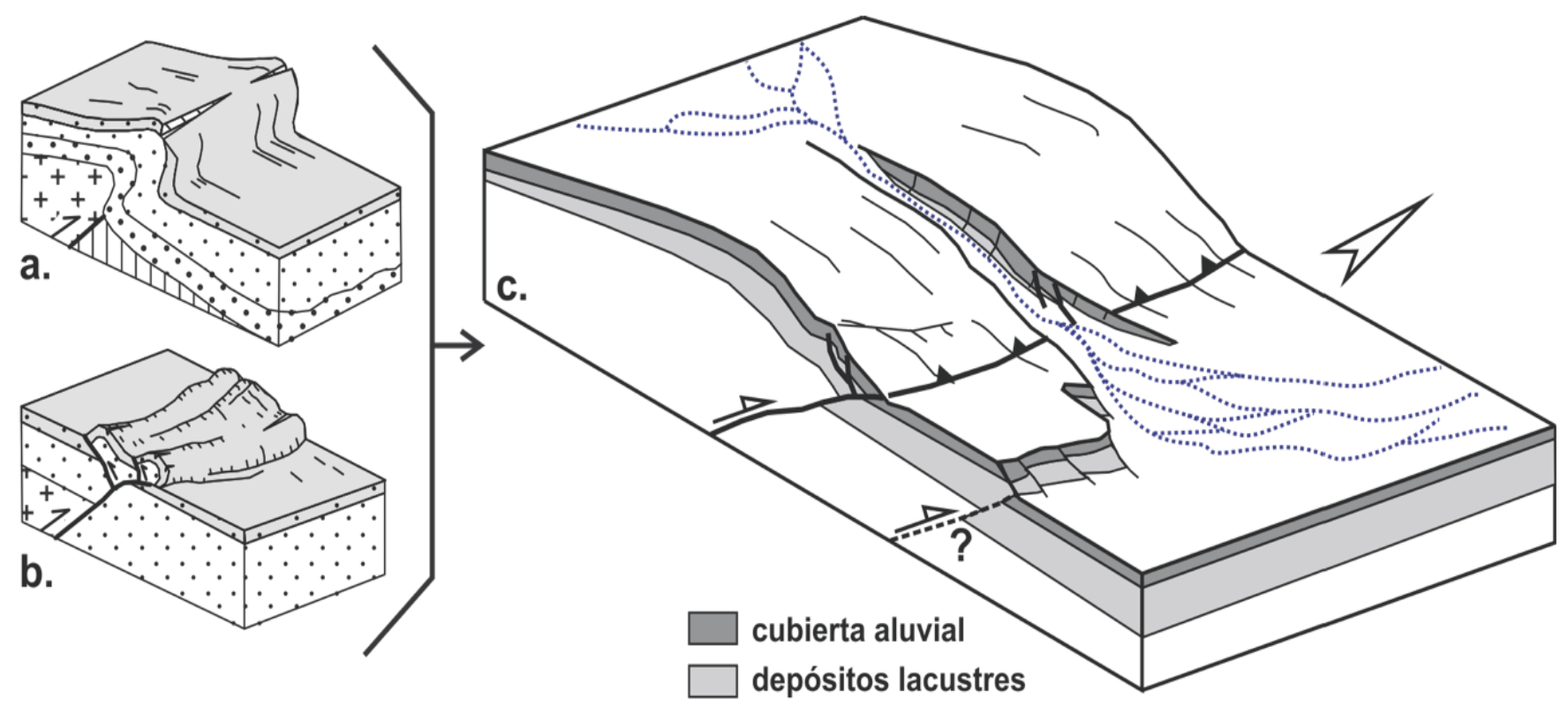

Figura 12 Modelo geomorfológico propuesto para la falla Acequión. a) Escarpa de limbo de pliegue. b) Escarpa de cresta de presión con retrocabalgamientos. a y b) tomados de Yu et al. (2010). c) Modelo de escarpa propuesto. Se combina una escarpa de flexura con una cresta de presión en la base, con retrocabalgamientos. Hacia el sur, nuevos cabalgamientos podrían afectar los depósitos cuaternarios más al este. 
un despegue común en profundidad. Una propuesta similar fue esbozada por Audemard et al. (2016) para quienes la falla Acequión solo sería una parte más de un sistema complejo de cabalgamientos, que incluso podría llegar a incluir a la falla $\mathrm{La}$ Chilca (Figura 2), también de vergencia andina, que afecta depósitos holocenos y cuya traza corre a los pies de la sierra de Pedernal, desdibujando, o más aun indicando el cierre de, la zona triangular coincidente con el corredor tectónico de Matagusanos-Maradona-Acequión, al menos a nivel superficial.

\subsection{ANÁLISIS TOPOGRÁFICO DE LA ESGARPA E IMPLICANGIAS PALEOSISMOLÓGICAS}

Se ha realizado un análisis topográfico de la escarpa de la falla Acequión, tomando como base metodologías propuestas por Carretier et al. (2002), Ewiak et al. (2015) y recientemente actualizadas por Wei et al. (2019). De los perfiles realizados, se obtuvo información sobre la altura y pendiente de la escarpa, el desplazamiento vertical de la superficie y los cambios en el gradiente de la escarpa. La altura de escarpa no fue utilizada como parámetro para el análisis dado que no es confiable relacionarla con los desplazamientos a lo largo de la falla (Yang et al., 2015). A partir del análisis estadístico se clasificaron los resultados según la cantidad de quiebres de pendiente identificados en cada perfil y los desplazamientos verticales medidos. Se observó que el agrupamiento por número de quiebres tuvo una gran dispersión en todos los casos, probablemente dada una posible degradación del perfil de escarpa, más aún en aquellos perfiles con mayor altura de escarpa (Tabla 1; Figura 10a). Modelos digitales de elevación de mayor resolución horizontal podrían ayudar a discernir este punto. A su vez, dado el modelo de escarpa propuesto (sección 5.1), se podría llegar a interpretar que algunos de los quiebres de pendiente corresponden a crestas de presión causadas tras distintos eventos, lo cual suma una nueva complejidad al análisis paleosismológico a partir del número de quiebres de pendiente.

Por otra parte, habiéndose interpretado como una escarpa flexural y, observando la distribución de desplazamientos verticales de la superficie, se puede llegar a obtener información a partir del agrupamiento por desplazamiento vertical. Según Wei et al. (2019), la anterior es una forma válida de cuantificar el desplazamiento acumulado a lo largo de una misma estructura, particularmente útil en ambientes donde ciclos geomórficos garanticen una tasa de formación de niveles guías superior a la tasa de recurrencia sísmica. Particularmente la Pampa de Acequión muestra dos niveles de terrazas holocenas y entre dos y tres niveles lacustres del Pleistoceno tardío a Holoceno (Perucca et al., 2012; Audemard et al., 2016), cumpliendo dicho criterio.

Se repitió el procedimiento tres veces, de las cuáles sólo se presentan en este trabajo a fines ilustrativos, los resultados del experimento 1 (Figura 10). En total, se obtuvieron entre cinco y seis eventos posibles (Tabla 2), sin embargo, los valores correspondientes al evento 4 no mostraron buena superposición entre cada experimento, por lo que no se tuvo en cuenta. Luego se calcularon los valores de desplazamiento vertical acumulado promedio a partir de los tres experimentos para luego obtener el desplazamiento vertical correspondiente a cada único evento (Tabla 3). Así, tomando como dato de entrada valores medidos en afloramiento de $24^{\circ}$ y $33^{\circ}$ para la inclinación del plano de falla, se convirtieron los valores de desplazamiento vertical (DV) por evento en desplazamiento real (DR) sobre el plano de falla (Tabla 3). Todos los valores obtenidos de desplazamiento real mostraron incertidumbres elevadas (alrededor de $\pm 1 \mathrm{~m}$ ) por lo que, a modo de aproximación, para estimar los sismos potenciales se tomaron los valores mínimos de cada desplazamiento real. Siguiendo las relaciones de Wells y Coppersmith (1994), se obtuvieron las magnitudes potenciales de los 4/5 sismos posibles (Tabla 3). Las magnitudes mínimas varían entre Mw 6.5 y Mw 7.1. Estos valores son levemente mayores a la $\mathrm{Mw} 6.3 \pm 0.4$ que se puede estimar considerando los $12 \mathrm{~km}$ de longitud de la escarpa de falla Acequión como una posible ruptura superficial única. Cabe destacar que estos valores son seguramente mínimos ya que las escarpas a favor de la pendiente regional 
Tabla 2. Desplazamiento vertical acumulado por evento en los 3 experimentos (el experimento 1 corresponde al que se muestra en el trabajo).

\begin{tabular}{|l|c|c|c|c|c|c|c|}
\hline Eventos & & $\mathbf{1}$ & $\mathbf{2}$ & $\mathbf{3}$ & $\mathbf{4}$ & $\mathbf{5}$ & $\mathbf{6}$ \\
\hline Experimento 1 & & & & & & \\
\hline & & & & & & \\
\hline Agrupamiento por DV & DV & 1.3 & 3.5 & & 5.7 & 8.1 & 10.7 \\
\hline Experimento 2 & $\pm \sigma$ & 0.4 & 0.35 & & 0.35 & 0.4 & 0.6 \\
\hline Agrupamiento por DV & DV & 1.3 & 3.1 & 4.7 & & 8.1 & 11.3 \\
\hline Experimento 3 & $\pm \sigma$ & 0.4 & 0.3 & 0.4 & & 0.4 & 0.5 \\
\hline Agrupamiento por DV & DV & 1 & 3 & 4.5 & 6.6 & 8.3 & 10.7 \\
\hline
\end{tabular}

DV: desplazamiento vertical de la superficie.

son rápidamente erosionadas y/o cubiertas por el material aluvial holoceno. Las terrazas aluviales y los depósitos lacustres involucrados en la deformación guardan edades variables entre el Pleistoceno superior $(\sim 35 \mathrm{ka})$ y el Holoceno (Perucca et al., 2012). En particular, según Audemard et al. (2016), las terrazas aluviales sobre las que se forma la escarpa de falla son post-UMG $(\sim 14 \mathrm{ka})$. Sobre la base de estas edades es posible interpretar que estos eventos tienen tiempos de recurrencia cercanos a los $2 \mathrm{ka}$. Ahora bien, dada la ausencia de edades absolutas de estos niveles aluviales, se puede optar por una aproximación más conservadora y tener en cuenta las edades más antiguas como tope máximo de la deformación ( 35 ka), lo cual resultaría en tiempos de retorno del orden de los 6 ka.

A partir del análisis de dos cuñas coluviales identificadas en el afloramiento del sector sur (Figura 7d), Audemard et al. (2016) interpretaron al menos dos eventos asociados a la falla Acequión con tiempos de recurrencia de $6 \pm 2$ ka y magnitudes Mw 7 o mayores. Restan estudios detallados en las trincheras naturales presentes en el área para determinar el número exacto de eventos, pero la aproximación utilizada en este trabajo acerca magnitudes levemente menores a las proporcionadas por dichos autores. Previamente, Perucca y Moreiras (2008) y Perucca et al. (2009) habían determinado a partir del análisis de avalanchas de rocas y deformación en los depósitos lacustres que al menos cinco sismos habrían ocurrido en la región. Sin embargo, adjudicaron dichos eventos a la falla Cerro Salinas, ubicada más al este. En línea con los trabajos que se realizaron posteriormente, el estudio aquí presentado sugiere que la actividad neotectónica en la Pampa del Acequión se remonta al Pleistoceno tardío y hasta cinco sismos pudieron haber ocurrido en esta zona.

Se puede observar que estos parámetros, derivados mayormente de la clasificación estadística de desplazamientos verticales, permiten estimar 
Tabla 3. Valores finales a partir de los tres experimentos. En cursivas, los valores mínimos considerados para el cálculo de los sismos potenciales y en negrita los resultados finales.

\begin{tabular}{|c|c|c|c|c|c|c|c|}
\hline Eventos & & 1 & 2 & 3 & $4 *$ & 5 & 6 \\
\hline \multicolumn{8}{|l|}{ Totales } \\
\hline \multirow{2}{*}{$\begin{array}{l}\text { DV acumulado, promediado } \\
\text { entre los } 3 \text { experimentos }\end{array}$} & DV & 1.2 & 3.2 & 4.6 & & 8.2 & 10.9 \\
\hline & $\pm \sigma$ & 0.4 & 0.3 & 0.3 & & 0.4 & 0.6 \\
\hline \multirow[t]{4}{*}{ DV por evento (m) } & DV & 1.2 & 2.0 & 1.4 & & 2.0 & 2.7 \\
\hline & $\pm \sigma$ & 0.4 & 0.3 & 0.3 & & 0.3 & 0.5 \\
\hline & DV max & 1.6 & 2.3 & 1.7 & & 2.4 & 3.2 \\
\hline & $\mathrm{DV} \min$ & 0.8 & 1.7 & 1.1 & & 1.7 & 2.2 \\
\hline \multirow[t]{4}{*}{ DR por evento (m) } & DR max & 3.5 & 5.2 & 3.8 & & 5.3 & 7.2 \\
\hline & $D R \min$ & 1.4 & 2.9 & 1.9 & & 2.9 & 3.8 \\
\hline & DR & 2.5 & 4.0 & 2.8 & & 4.1 & 5.5 \\
\hline & $\pm \sigma$ & 1.0 & 1.2 & 0.9 & & 1.2 & 1.7 \\
\hline \multicolumn{8}{|c|}{ Magnitudes Potenciales Mínimas } \\
\hline & $M w$ & 6.6 & 6.7 & 6.6 & & 6.7 & 6.8 \\
\hline & $\pm \sigma$ & 0.1 & 0.2 & 0.2 & & 0.2 & 0.3 \\
\hline \multicolumn{8}{|c|}{ *evento 4 no considerado para este análisis (ver texto). } \\
\hline
\end{tabular}

de manera aproximada el comportamiento paleosísmico de una estructura. Pueden ser relativamente confiables en zonas de baja complejidad tectónica (Yang et al., 2015), lo cual puede no necesariamente ser el caso de la zona de trabajo, donde se encuentran dos estilos estructurales diferentes. Los valores obtenidos en este estudio con otros previamente publicados para la zona muestran una relativa buena correlación que deberá ser constatada con análisis paleosismológicos de detalle en las exposiciones naturales y dataciones absolutas de las superficies aluviales involucradas en la deformación. Metodologías como modelados numéricos, tomando como datos de entrada los ángulos de falla y la topografía también pueden resultar útiles en estos casos para estimar parámetros sismogénicos (Hardy y Allmendinger, 2011; Vázquez et al., 2016; Colavitto et al., 2020). 


\section{Conclusiones}

En este trabajo se analizaron las características de una escarpa de falla inversa en el sur de la provincia de San Juan, Argentina. Este rasgo se asocia a la actividad tectónica cuaternaria (Pleistoceno superior-Holoceno) de la falla Acequión. Estudios anteriores indicaron la ocurrencia de al menos dos eventos de $\mathrm{Mw}>7$ asociados a esta estructura. Se presentaron en este trabajo los resultados de un análisis topográfico de la escarpa, estudiando estadísticamente cambios en los desplazamientos verticales y quiebres de pendiente a lo largo de una escarpa. Se realizaron 70 perfiles transversales a lo largo de la escarpa de falla de al menos 12 $\mathrm{km}$ de extensión, de los cuales aproximadamente la mitad presentó un solo quiebre de pendiente (un evento potencial) y menos del 10\% mostraron cuatro quiebres de pendiente (cuatro eventos potenciales). A la vez se agruparon los perfiles obtenidos según el desplazamiento vertical observado, en cuyo caso se pudieron identificar hasta cinco eventos potenciales. Bajo esta clasificación, se estimaron las magnitudes potenciales mínimas de dichos eventos, que varían entre $\mathrm{Mw} 6.5$ y Mw 7.1. Se estudiaron además evidencias de campo que permitieron interpretar que la falla Acequión produjo una escarpa flexural con cresta de presión con retrocabalgamientos. Esta interpretación permite incluir a esta estructura dentro del dominio andino. A su vez, un levantamiento gravimétrico de detalle, acompañado del estudio de modelos digitales de elevación, permitió identificar dos estructuras subparalelas y en posición más oriental a la falla Acequión, que podrían sugerir un avance de la deformación hacia el este, al menos en el sector sur de la Pampa del Acequión tal como fue sugerido en trabajos previos, en los que se identificaron fallas holocenas sobre el frente de la sierra de Pedernal. Es necesario continuar estudiando las exposiciones naturales del área a los fines de contrastar las conclusiones obtenidas en este trabajo y así poder refinar la historia paleosísmica de esta estructura, cuyo potencial sismogénico podría ser importante para la región del sur de San Juan y norte de Mendoza.

\section{Agradecimientos}

La presente contribución fue financiada con fondos de los proyectos PID CICITCA 2018-19 (Universidad Nacional de San Juan), PID 013 del Consejo Nacional de Investigaciones Científicas y Técnicas (CONICET), PICT 2016/0995 FONCYT and IPGH (GEOF 03-18). Los autores y autoras agradecen a la Asociación Argentina de Cuaternario y Geomorfología por la ayuda económica que permitirá continuar con los estudios paleosismológicos. También se agradecen las revisiones realizadas por Víctor Ramos y Luz Rodríguez que ayudaron a mejorar el manuscrito final.

\section{Referencias}

Ahumada, E. A., 2010, Neotectónica del Frente Orogénico Andino entre los $32^{\circ} 08^{\prime}$ S-32 ${ }^{\circ} 19^{\prime}$ $\mathrm{S}$, provincias de Mendoza y San Juan: San Luis, Argentina, Universidad Nacional de San Luis, tesis doctoral, $155 \mathrm{p}$.

Ahumada, E. A., Costa, C. H., 2009, Antithetic linkage between oblique Quaternary thrusts at the Andean front, Argentine Precordillera: Journal of South American Earth Sciences, 28, 207-216. https://doi.org/10.1016/j. jsames.2009.03.008

Alcacer Sánchez, J. M., Perucca, L. P., 2018, Análisis neotectónico en el piedemonte oriental de Cordillera Frontal, Andes Centrales. San Juan, Argentina: Geociências, 36, 691-702.

Anderson, M., Alvarado, P., Zandt, G., Beck, S., 2007, Geometry and brittle deformation of the subducting Nazca Plate, Central Chile and Argentina: Geophysical Journal International, 171, 419-434. https://doi. org/10.1111/j.1365-246X.2007.03483.x

Andrews, D., Hanks, T., 1985, Scarp degraded by linear diffusion: Inverse solution for age: Journal of Geophysical Research: Solid Earth, 90, 10193-10208. https://doi. org/10.1029/JB090iB12p10193 
Arrowsmith, J. R., Pollard, D. D., Rhodes, D. D., 1996, Hillslope development in areas of active tectonics: Journal of Geophysical Research: Solid Earth, 101, 6255-6275. https://doi.org/10.1029/95JB02583

Audemard, F. A., 1999, Morpho-structural expression of active thrust fault systems in the humid tropical foothills of Colombia and Venezuela: Zeitschrift für Geomorphologie. Supplementband, 118, 227-244.

Audemard, F. A., 2003, Geomorphic and geologic evidence of ongoing uplift and deformation in the Mérida Andes, Venezuela: Quaternary International, 101, 43-65. https://doi. org/10.1016/S1040-6182(02)00128-3

Audemard, F. A., 2009, Flexura Frontal Surandina, Venezuela (VE-07). En Proyecto Mutinacional Andino. Geociencia para las comunidades andinas (eds.), Atlas de deformaciones cuaternarias de Los Andes. Publicación Geológica Multinacional, 7, 300-311.

Audemard, F. A., Perucca, L. P., Pantano Zuñiga, A. V., Ávila, C. R., Onorato, M. R., Vargas, H. N., Alvarado, P., Viete, H., 2016, Holocene compression in the Andes Precordillera, in the Acequión region (San Juan Province, Argentina): Geomorphic, (micro-and meso-) tectonic, and (direct and indirect) paleoseismic evidence: Journal of South American Earth and Sciences, 67, 140-157. https://doi.org/10.1016/j. jsames.2016.02.005

Baldis, B. A., Chebli, G., 1969, Estructura profunda del área central de la Precordillera sanjuanina: Jornadas Geológicas Argentinas, 1, 47-65.

Bastías, H., Weidmann, N., Pérez, M., 1984, Dos zonas de fallamiento Plio-Cuaternario en la Precordillera de San Juan, en $9^{\circ}$ Congreso Geológico Argentino, Bariloche, Río Negro, Argentina, Asociación Geológica Argentina, 329-341.

Bucknam, R., Anderson, R., 1979, Estimation of fault-scarp ages from a scarp-heightslope-angle relationship: Geology, 7,
11-14. https://doi.org/10.1130/00917613(1979)7<11:EOFAFA>2.0.CO;2

Burbank, D. W., Anderson, R. S., 2011, Tectonic geomorphology. John Wiley y Sons.

Cahill, T., Isacks, B. L., 1992, Seismicity and shape of the subducted Nazca plate: Journal of Geophysical Research: Solid Earth, 97, 17503-17529. https://doi. org/10.1029/92JB00493

Carretier, S., Ritz, J., Jackson, J., Bayasgalan, A., 2002, Morphological dating of cumulative reverse faultscarps: examplesfrom the Gurvan Bogd fault system, Mongolia: Geophysical Journal International, 148, 256-277. https:/ / doi.org/10.1046/j.1365-246X.2002.01599.x

Colavitto, B., Sagripanti, L., Jagoe, L., Costa, C. H., Folguera, A., 2020, Quaternary tectonics in the southern Central Andes $\left(37^{\circ}-38^{\circ}\right.$ S): Retroarc compression inferred from morphotectonics and numerical models: Journal of South American Earth Sciences, 102, 102697. https://doi.org/10.1016/j. jsames.2020.102697

Cortés, J. M., Casa, A. L., Pasini, M. M., Yamin, M. G., Terrizzano, C. M., 2006, Fajas oblicuas de deformación neotectónica en Precordillera y Cordillera Frontal $\left(31^{\circ} 30^{\prime}-33^{\circ}\right.$ 30' LS): controles paleotectónicos: Revista de la Asociación Geológica Argentina, 61, 639-646

Costa, C. H., Rockwell, T., Paredes, J., Gardini, C., 1999, Quaternary deformations and seismic hazard at the Andean orogenic front $\left(31^{\circ}-33^{\circ}\right.$, Argentina): A paleoseismological perspective, en $4^{\text {th }}$ International Symposium on Andean Geodynamics, Extended abstracts, París, 187-191.

Costa, G. H., Audemard, F., Bezerra, F. H., Lavenu, A., Machette, M. N., París, G., 2006, Una perspectiva sobre las principales deformaciones cuaternarias de América del Sur: Revista de la Asociación Geológica Argentina, 61, 461-479.

Costa, C. H., Alvarado, A., Audemard, F. A., Audin, L., Benavente, C., Bezerra, F. H., Cembrano, J., González, G., López, M., 
Minaya, E., Santibañez, I., García, J., Arcila, M., Pagani, M., Pérez, I., Delgado, F., Paolini, M., Garro, H., 2020, Hazardous faults of South America; compilation and overview: Journal of South American Earth Sciences, 102837. https://doi.org/10.1016/j. jsames.2020.102837

Ewiak, O., Victor, P., Oncken, O., 2015, Investigating multiple fault rupture at the Salar del Carmen segment of the Atacama Fault System (northern Chile): Fault scarp morphology and knickpoint analysis: Tectonics, 34, 187-212. https://doi. org/10.1046/j.1365-246X.2002.01599.x

Farr, T. G., Rosen, P. A., Caro, E., Crippen, R., Duren, R., Hensley, S., Kobrick, M., Paller, M., Rodriguez, E., Roth, L., 2007, The shuttle radar topography mission: Reviews of Geophysics, 45(2), 1-33. https://doi. org/10.1029/2005RG000183

Giampaoli, P., Cegarra, M. I., 2003, Análisis estructural del extremo sur de la Precordillera Central sanjuanina: Revista de la Asociación Geológica Argentina, 58, 49-60.

Hardy, S., Allmendinger, R. W., 2011, Trishear: A review of kinematics, mechanics, and applications, en K. McClay, J. Shaw, and J. Suppe (eds.), Thrust fault-related folding: AAPG Memoir 94, 95-119. https://doi. org/10.1306/13251334M943429

Heim, A., 1952, Estudios tectónicos en la Precordillera de San Juan: los ríos San Juan, Jáchal y Huaco: Revista de la Asociación Geológica Argentina, 7, 11-70.

Hinze, W. J., 2003, Bouguer reduction density, why 2.67?: Geophysics, 68, 1559-1560. https:// doi.org/10.1190/1.1620629

Jordan, T. E., Isacks, B. L., Ramos, V. A., Allmendinger, R. W., 1983, Mountain building in the Central Andes: Episodes, 3, 20-26.

Kane, M., 1962, A comprehensive system of terrain corrections using a digital computer: Geophysics, 27, 455-462. https://doi. org/10.1190/1.1439044
Klinger, Y., Etchebes, M., Tapponnier, P., Narteau, C., 2011, Characteristic slip for five great earthquakes along the Fuyun fault in China: Nature Geoscience, 4, 389-392. https://doi. org/10.1038/ngeol 158

Lara, G., Perucca, L. P., Rothis, M., Pantano Zuñiga, A. V., Sáez, M., 2018, Actividad tectónica cuaternaria del Sistema de Falla Maradona, Precordillera Central, Argentina: Andean Geology, 45, 145-160. https://doi. org/10.5027/andgeov45n2-2970

Litchfield, N. J., Campbell, J. K., Nicol, A., 2003, Recognition of active reverse faults and folds in North Canterbury, New Zealand, using structural mapping and geomorphic analysis: New Zealand Journal of Geology and Geophysics, 46, 563-579. https://doi.or $\mathrm{g} / 10.1080 / 00288306.2003 .9515030$

López, M., Audemard, F. A., Velasquez, A., 2011, Paleoseismic evidence of Holocene compression at Tulua in the valle del Cauca, along the west foothills of the central cordillera of Colombia. En Audemard, F. A., Michetti A. M., McCalpin,J. (eds.) Geological criteria for evaluating seismicity revisited: 40 years of paleoseismic investigations and the natural record of past earthquakes, GSA Special papers, 479, 91-107. https://doi. org/10.1130/2011.2479(04).

Mayer, L., 1984, Dating Quaternary fault scarps formed in alluvium using morphologic parameters: Quaternary Research, 22, 300-313. https://doi. org/10.1016/0033-5894(84)90024-3

McCalpin, J. P., 2009, Paleoseismology. Academic press.

McGill, S. F., Sieh, K., 1991, Surficial offsets on the central and eastern Garlock fault associated with prehistoric earthquakes: Journal of Geophysical Research: Solid Earth, 96, 21597-21621. https://doi. org/10.1029/91JB02030

Mériaux, A.S., Ryerson, F., Tapponnier, P., Van der Woerd, J., Finkel, R., Xu, X., Xu, Z., Caffee, M., 2004, Rapid slip along the central 
Altyn Tagh Fault: morphochronologic evidence from Cherchen $\mathrm{He}$ and Sulamu Tagh: Journal of Geophysical Research: Solid Earth, 109(B6), B06401. https://doi. org/10.1029/2003JB002558

Nagy, D., 1966, The gravitational attraction of a right rectangular prism: Geophysics, 31, 362371. https://doi.org/10.1190/1.1439779

Ollarves, R., Audemard, F. A., López, M., 2006, Morphotectonic Criteria for the Identification of Active Blind Faulting in Alluvial Environments: Case Studies from Venezuela and Colombia. En Latrubesse, E. (ed.) Tropical Geomorphology with special emphasis on South America. Zeitschrift für Geomorphologie, 145, 81-103

Ortiz, A., Zambrano, J., 1981, La provincia geológica Precordillera oriental, en $8^{\circ}$ Congreso Geológico Argentino, San Luis, Argentina, Actas 3, 59-74.

Pantano Zuñiga, A. V., 2015, Geomorfología, neotectónica y peligrosidad geológica en la cuenca del río Acequión, provincia de San Juan, Argentina: Tucumán, Argentina, Universidad Nacional de Tucumán, tesis doctoral, $161 \mathrm{p}$.

Paredes, J., Perucca, L. P., 2000, Fallamiento cuaternario en la depresión de Matagusanos, San Juan, Argentina: Revista de la Asociación Geológica Argentina, 55, 23.

Peri, V. G., Rapalini, A. E., Pérez, P., Franceschinis, P., Leiva, F., Fazzito, S. Y., Cortés, J. M., 2020, Subsurface characterization of quaternary scarps and their possible connection to main structures of the western margin of Precordillera, San Juan, Argentina: Tectonophysics, 790, 228542. https://doi. org/10.1016/j.tecto.2020.228542

Perucca, L. P., 1990, Sistema de fallamiento La Dehesa-Maradona-Acequión, San Juan, Argentina, En $11^{\circ}$ Congreso Geológico Argentino: San Juan, Argentina, Asociación Geológica Argentina, Actas 2, 431-434.

Perucca, L. P., Moreiras, S. M., 2006, Liquefaction phenomena associated with historical earthquakes in San Juan and
Mendoza Provinces, Argentina: Quaternary International, 158, 96-109. https://doi. org/10.1016/j.quaint.2006.05.023

Perucca, L. P., Moreiras, S. M., 2008, Indicative structures of paleoseismicity in the Acequion river valley, San Juan province, centralwestern Argentina: Geodinamica Acta, 21, 93-105. https://doi.org/10.3166/ ga.21.93-105

Perucca, L. P., Onorato, M. R., 2011, Fallas con actividad cuaternaria en el corredor tectónico Matagusanos-Maradona-Acequión entre los ríos de La Flecha y del Agua. Provincia de San Juan: Revista de la Asociación Geológica Argentina, 68, 38-51.

Perucca, L. P., Vargas, H. N., 2014, Neotectónica de la provincia de San Juan, centro-oeste de Argentina: Boletín de la Sociedad Geológica Mexicana, 66, 291-304. https://doi. org/10.18268/bsgm2014v66n2a6

Perucca, L. P., Bracco, A. I., Moreiras, S. M., 2009, Determination of seismogenic structures and earthquake magnitud from seismites in the Acequion river, Precordillera Range, centralwestern Argentina/Determinación de las estructuras sismogénicas y la magnitud del terremoto a partir de sismitas en el río Acequión, Precordillera, centro-oeste argentino: Journal of Iberian Geology, 35, 5-18.

Perucca, L. P., Audemard, F. A., Pantano Zuñiga, A. V., Vargas, H. N., Ávila, C. R., Onorato, M. R., 2012, Vergencias opuestas cuaternarias en el área de Acequión, Precordillera de San Juan (Argentina): Revista de la Sociedad Geológica de España, 25, 5-15.

Perucca, L. P., Audemard, F. A., Pantano Zuñiga, A. V., Vargas, H. N., Avila, C. R., Onorato, M. R., Lara, G., Esper Angillieri, M. Y., 2013, Fallas cuaternarias con vergencias opuestas entre Precordillera Central y Oriental, provincia de San Juan: Revista de la Asociación Geológica Argentina, 70, 291-302.

Perucca, L. P., Godoy, E., Pantano Zuñiga, A. V., 2014a, Late Pleistocene-Holocene earthquakeinduced slumps and soft-sediment 
deformation structures in the Acequion River valley, Central Precordillera, Argentina: Geologos, 20, 147-156. https://doi. org/10.2478/logos-2014-0007

Perucca, L. P., Onorato, M. R., Millán, J., Bustos, A., Vargas, H. N., 2014b, Variación del estilo de deformación a lo largo del Sistema de falla La Cantera, Precordillera Central, San Juan, Argentina: Revista de la Sociedad Geológica de España, 27, 69-79.

Perucca, L. P., Rothis, M., Bezerra, F. H., Vargas, H. N., Lima, J., 2015, Late quaternary evolution of the La Cantera Fault System (Central Precordillera, Argentina): A morphotectonic and paleoseismic analysis: Tectonophysics, 661, 200-209. https://doi. org/10.1016/j.tecto.2015.08.

Philip, H., Rogozhin, E., Cisternas, A., Bousquet, J., Borisov, B., Karakhanian, A., 1992, The Armenian earthquake of 1988 December 7: faulting and folding, neotectonics and palaeoseismicity: Geophysical Journal International, 110, 141-158. https://doi. org/10.1111/j.1365-246X.1992.tb00718.x

Ramos, V. A., 1988, The tectonics of the Central Andes; $30^{\circ}$ to $33^{\circ} \mathrm{S}$ latitude, En Clark,S.P., Burchfiel, B. C., Suppe, J.(Eds.), Processes in continental lithospheric deformation, GSA Special Papers, 218, 31.

Ramos, V. A., 1997, El Segmento de Subducción Subhorizontal de los Andes Centrales ArgentinosChilenos: Acta geológica hispánica, 5-16.

Ramos, V. A., 1999, Las provincias geológicas del territorio argentino, En Caminos, R., (Ed.), Geología Argentina, Instituto de Geología y Recursos Minerales, Anales Buenos Aires.

Ramos, V. A., Cristallini, E. O., Pérez, D. J., 2002, The Pampean flat-slab of the Central Andes: Journal of South American Earth Sciences, 15, 59-78. https://doi.org/10.1016/ S0895-9811(02)00006-8

Ren, Z., Zhang, Z., Chen, T., Yan, S., Yin, J., Zhang, P., Zheng, W., Zhang, H., Li, C., 2016, Clustering of offsets on the Haiyuan fault and their relationship to paleoearthquakes: Bulletin, 128, 3-18. https://doi.org/10.1130/B31155.1

Siame, L. L., Sébrier, M., Bellier, O., Bourlés, D., Castano, J. C., Araujo, M., 1997, Geometry, segmentation and displacement rates of the El Tigre Fault, San Juan Province (Argentina) from SPOT image analysis and 10Be datings: Annales Tectonicae.

Siame, L. L., Bellier, O., Sébrier, M., 2006, Active tectonics in the Argentine Precordillera and western Sierras Pampeanas: Revista de la Asociación Geológica Argentina, 61, 604-619.

Smalley, R., Pujol, J., Regnier, M., Chiu, J.M., Chatelain, J. L., Isacks, B. L., Araujo, M., Puebla, N., 1993, Basement seismicity beneath the Andean Precordillera thinskinned thrust belt and implications for crustal and lithospheric behavior: Tectonics, 12, 6376. https://doi.org/10.1029/92TC01108

Somigliana, C., 1930, Sul campo gravitazionale esterno del geoide ellissoidico: Atti della Reale Accademia Nazionale dei Lincei, Rendiconti 6, 237-243.

Tassara, A., Echaurren, A., 2012, Anatomy of the Andean subduction zone: three-dimensional density model upgraded and compared against global-scale models: Geophysical Journal International, 189, 161-168. https:// doi.org/10.1111/j.1365-246X.2012.05397.x

Terrizzano, C. M., Fazzito, S. Y., Cortés, J. M., Rapalini, A. E., 2010, Studies of Quaternary deformation zones through geomorphic and geophysical evidence: A case in the PrecordilleraSur, CentralAndes of Argentina: Tectonophysics, 490, 184-196. https://doi. org/10.1016/j.tecto.2010.05.006

Terrizzano, C.M., Cortés, J. M.,Zech, R., Morabito García, E., 2014, Drainage anomalies as indicators of soft-linked deformation zones between neotectonic structural highs in the Precordillera, Central Andes of Argentina: Geomorphology, 224, 1-15. https://doi. org/10.1016/j.geomorph.2014.07.004

Vázquez, F. R., Costa, G. H., Gardini, C. E., 2016, Quaternary-active thrusts scarps 
tested as deformation markers by trishear models in the Southern Precordillera of Argentina: Quaternary International, 438, 65-79. https://doi.org/10.1016/j. quaint.2016.06.020

Wallace, R. E., 1968, Notes on stream channels offset by the San Andreas fault, southern Coast Ranges, California, en Conference on Geologic Problems of the San Andreas Fault System. Stanford University Publication in Geological Sciences.

Wallace, R. E., 1977, Profiles and ages of young fault scarps, north-central Nevada: Geological Society of America Bulletin, 88, 1267-1281. https://doi.org/10.1130/0016$7606(1977) 88<1267: \mathrm{PAAOYF}>2.0$. $\mathrm{CO} ; 2$

Wei, Z., He, H., Su, P., Zhuang, Q., Sun, W., 2019, Investigating paleoseismicity using fault scarp morphology of the Dushanzi Reverse Fault in the northern Tian Shan, China: Geomorphology, 327, 542-553. https://doi. org/10.1016/j.geomorph.2018.11.025

Wells, D. L., Coppersmith, K. J., 1994, New empirical relationships among magnitude, rupture length, rupture width, rupture area, and surface displacement: Bulletin of the seismological Society of America, 84, 974-1002.

Yang, X., Li, W., Qin, Z., 2015, Calculation of reverse-fault-related parameters using topographic profiles and fault bedding: Geodesy and Geodynamics, 6, 106-112. https://doi.org/10.1016/j.geog.2014.09.002

Yu, G., Xu, X., Klinger, Y., Diao, G., Chen, G., Feng, X., Li, C., Zhu, A., Yuan, R., Guo, T., Sun, X., Tan, X., An, Y., 2010, Fault-scarp features and cascading-rupture model for the $\mathrm{M} w$ 7.9 Wenchuan earthquake, eastern Tibetan plateau, China: Bulletin of the Seismological Society of America, 100, 2590-2614. https:// doi.org/10.1785/0120090255

Zapata, T. R., Allmendinger, R. W., 1996, Thrust-front zone of the Precordillera, Argentina: a thickskinned triangle zone: AAPG Bulletin, 80, 359-381. https://doi. org/10.1306/64ED87E6-1724-11D78645000102C1865D

Zielke, O., Arrowsmith, J. R., Ludwig, L. G., Akciz, S. O., 2012, High-Resolution TopographyDerived Offsets along the 1857 Fort Tejon Earthquake Rupture Trace, San Andreas FaultHighResolution TopographyDerived Offsets along the 1857 Fort Tejon Rupture, San Andreas Fault: Bulletin of the Seismological Society of America, 102, 11351154. https://doi.org/10.1038/ngeo1 158

Zielke, O., Klinger, Y., Arrowsmith,J. R., 2015, Fault slip and earthquake recurrence along strikeslip faults - Contributions of high-resolution geomorphic data: Tectonophysics, 638, 43-62. https://doi.org/10.1016/j.tecto.2014.11.004 